\title{
MINIMIZING VARIATIONAL CURVES RESTRICTED TO A PREASSIGNED SET
}

\author{
BY
J. WARGA
}

I. Introduction. The purpose of the present paper is to derive "constructive" necessary conditions for minimum in problems of the calculus of variations in which the "controls" are chosen from a compact Hausdorff space (in particular, a compact euclidean set) and the admissible curves are restricted to lie in a given closed set with a smooth boundary and must satisfy given boundary conditions.

Let $R$ be a compact Hausdorff space, $E_{n}$ the euclidean $n$-space, $T$ the closed interval $\left[t_{0}, t_{1}\right]$ of the real axis, $V$ an open set in $E_{n}$ and $B_{0}$ and $B_{1}$ closed sets in $V$. We also assume given a function $g(x, t, \rho)=\left(g^{1}(x, t, \rho), \cdots, g^{n}(x, t, \rho)\right)$ from $V \times T \times R$ to $E_{n}$ and a scalar (real-valued) function $a(x)$ defined over $V$.

Let $G(x, t)=\{g(x, t, \rho) \mid \rho \in R\}, x \in V, t \in T$, and let $F(x, t)$ be the convex closure of $G(x, t)$.

We define an original admissible curve with respect to $a(x)$ as any absolutely continuous function $x(t)$ from $T$ to $V$ such that, for some function $\rho(t)$ from $T$ to $R$,

$$
\frac{d x(t)}{d t}=\dot{x}(t)=g(x(t), t, \rho(t)) \text { a.e. in } T
$$

or, equivalently,

$$
\dot{x}(t) \in G(x(t), t) \text { a.e. in } T
$$

and

$$
\begin{aligned}
x\left(t_{0}\right) \in B_{0}, & x\left(t_{1}\right) \in B_{1}, \\
a(x(t)) & \leqq 0, \quad t \in T .
\end{aligned}
$$

A relaxed admissible curve with respect to $a(x)$ is similarly defined except that the relation (1.1) resp. (1.1 Orig) is replaced by

(1.1 Relaxed)

$$
\dot{x}(t) \in F(x(t), t) \text { a.e. in } T \text {. }
$$

An original (resp. relaxed) minimizing curve with respect to $a(x)$ is one

Received by the editors April 11, 1963. 
which minimizes, among all original (resp. relaxed) admissible curves with respect to $a(x)$, the value $x^{1}\left(t_{1}\right)$.

These definitions coincide with those of $[1$, p. 112] and [2, p. 129] in the special case when the set $A$ of these references is defined by the inequality $a(x) \leqq 0$ and the set $B$ of the references is replaced by $B_{0} \times B_{1}$. However, we now dispense with the assumption on which the arguments of [2] are based, namely, that there exists a relaxed minimizing curve in the interior of $A$.

It has been shown by Young [3, p. 233], McShane [4, p. 527 and p. 532], Filippov [5, p. 76] and Warga $\left(^{1}\right)[1$, p. 119 and p. 123] that for certain classes of variational problems a relaxed minimizing curve with respect to $a(x)$ exists; furthermore [1, p. 113], a relaxed minimizing curve with respect to $a(x)$ can be uniformly approximated by solutions of the differential equations (1.1). On the other hand, Gamkrelidze $[6 ; 7$, p. 257] has derived necessary conditions which must be satisfied by original minimizing curves with respect to $a(x)$ in a certain class of problems, subject to the prior assumption that these curves are "regular," have piecewise smooth "controls" and a finite number of "junction points" [7, pp. 258, 265-266 and p. 311].

It is our purpose in this paper to investigate necessary conditions satisfied by relaxed minimizing curves with respect to $a(x)$ without making any a priori assumptions about these minimizing curves. We define, in $\S I$ II, a rather wide class of variational problems and prove, in Theorem 3.1, that there exists, in these problems, a relaxed minimizing curve with respect to $a(x)$, that this curve can be uniformly approximated by solutions of the differential equations (1.1), and that this curve satisfies necessary conditions which generalize the classical Weierstrass $E$-condition and transversality conditions (and the analogous conditions of Young [8], McShane [9], Pontryagin et al. [10], Warga [2] and Gamkrelidze [6; 7, p. 257]).

Added in proof. A more general problem, in which the admissible curves are restricted to satisfy the simultaneous inequalities $a^{k}(x(t)) \leqq 0, t \in T, k=1, \cdots, m$, instead of relation (1.3), has been considered and a corresponding generalization of Theorem 3.1 has been obtained. This more general problem will be discussed elsewhere.

II. Assumptions and definitions. We define the class of variational problems which are being considered as those which satisfy the following assumption:

Assumption 2.1. There exist a (Lebesgue) measurable subset $T^{\prime}$ of $T$ of measure $\left|T^{\prime}\right|=|T|$, positive constants $c_{1}$ and $\varepsilon_{1}$, an open set $V^{\prime} \subset V$ and a compact set $D \subset V^{\prime}$ such that

2.1.1. The functions $g^{i}(x, t, \rho)$ and $\partial g^{i}(x, t, \rho) / \partial x^{j}, i, j=1, \cdots, n$, exist over $V^{\prime} \times T^{\prime} \times R$ and, over that set, are continuous functions of $(x, t)$ uniformly in $\rho$,

(1) The proof of Theorem 3.1 of $[1, \mathrm{p} .119]$ is very similar to the proof of Filippov's Theorem 1 of [5, p. 76]. The author is indebted to L. W. Neustadt for drawing his attention to Filippov's paper which antedates [1] by approximately three years. 
are continuous functions $\left({ }^{2}\right)$ of $t$ uniformly in $(x, \rho)$, and are continuous functions of $\rho$ for each $(x, t)$.

2.1.2. $|g(x, t, \rho)| \leqq c_{1}$ and $\left|g_{x}(x, t, \rho)\right| \leqq c_{1}$ over $V^{\prime} \times T^{\prime} \times R$ (where $g_{x}$ represents the matrix $\left(\partial g^{i} / \partial x^{j}\right), i, j=1, \cdots, n,|g|$ is the euclidean length of $g$ and $\left.\left|g_{x}\right|=\sum_{i, j=1}^{n}\left|\partial g^{i} / \partial x^{j}\right|\right)$.

2.1.3. The scalar function $a(x)$ is continuous, has continuous first and second order partial derivatives over $V^{\prime}$ and $\left|a_{x}(x)\right||g(x, t, \rho)| \leqq c_{1}$ over $V^{\prime} \times T^{\prime} \times R$, where $a_{x}$ is the gradient of $a(x)$.

2.1.4. There exists at least one relaxed admissible curve with respect to $a(x)$.

2.1.5. All relaxed admissible curves with respect to $a(x)-\varepsilon_{1}$ are contained in $D$.

REMARK. Assumption 2.1 can be easily verified in the following two cases:

(a) There exist positive $\varepsilon_{1}$ and $c_{1}$ such that the set $D=\left\{x \in V \mid a(x) \leqq \varepsilon_{1}\right\}$ is compact; Assumptions 2.1.1, 2.1.2 and 2.1.3 are satisfied for some open subset $V^{\prime}$ of $V$ containing $D$; and there exists at least one relaxed admissible curve with respect to $a(x)$.

(b) $V=E_{n}$; one of the sets $B_{0}$ or $B_{1}$ is bounded; there exist positive $\varepsilon_{1}$ and $c_{1}$ and a positive, continuously differentiable function $V(x)$ over $E_{n}$ such that $\left|V_{x}(x) \cdot g(x, t, \rho)\right| \leqq c_{1} \cdot V(x)$ for all $(t, \rho) \in T \times R$ and for all $x$ such that $a(x) \leqq \varepsilon_{1}$; the set $D=\left\{x \in E_{n} \mid V(x) \leqq e^{c_{1}\left(t_{1}-t_{0}\right)} V(b)\right.$ for some $\left.b \in B^{\prime}\right\}$ is compact, where $B^{\prime}$ is either $B_{0}$ or $B_{1}$, whichever set is bounded; there exists at least one relaxed admissible curve with respect to $a(x)$; and Assumptions 2.1.1, 2.1.2 and 2.1.3 are satisfied for some open set $V^{\prime}$ containing $D$.

The proof that Assumption 2.1 is satisfied in the case (b) follows the reasoning of [5, p. 77]. In particular, following Filippov, we may consider $V(x)=|x|^{2}+1$.

Having specified the class of problems which we propose to investigate, we shall now consider a convenient form for describing them. In particular, we wish to write relation (1.1 Relaxed) in the form of a system of differential equations analogous to (1.1); and we wish to replace relation (1.2) by one involving convex sets only. We do so by introducing "proper representations" of $F(x, t)$, of $B_{0}$ and of $B_{1}$.

Definition 2.2. A function $f(x, t, \sigma)$ from $V^{\prime} \times T \times S$ to $E_{n}$ is a proper representation of $F(x, t)$ if

2.2.1. $F(x, t)=\{f(x, t, \sigma) \mid \sigma \in S\}, x \in V^{\prime}, t \in T$.

2.2.2. For every absolutely continuous curve $x(t)$ satisfying (1.1 Relaxed) there exists a function $\sigma(t)$ from $T$ to $S$ such that

$$
\dot{x}(t)=f(x(t), t, \sigma(t)) \text { a.e. in } T
$$

(2) It can be easily shown that this assumption implies the existence of a finite or denumerable collection of disjoint Lebesgue measurable subsets $T_{r}$ of $T^{\prime}, r=1,2, \cdots$, and of a bounded $\varepsilon(h)$ converging to 0 with $h$ such that $\left|\bigcup T_{r}\right|=|T|$ and $\left|g^{i}(x, t, \rho)-g^{i}(x, \tau, \rho)\right| \leqq \varepsilon(t-\tau)$ for $(x, \rho) \in V^{\prime} \times R, t \in T_{r}, \tau \in T_{r}, i=1, \cdots, n, r=1,2, \cdots$. This last condition will be required whenever we shall refer to [1, Theorem 2.2, p. 113]. 
and $f(x, t, \sigma(\tau))$ is, for all $x \in V^{\prime}, t \in T^{\prime}$, a (Lebesgue) measurable function of $\tau$ over $T$.

2.2.3. $f^{i}(x, t, \sigma)$ and $\partial f^{i}(x, t, \sigma) / \partial x^{j}, i, j=1, \cdots, n$, exist and are continuous functions of $(x, t)$ over $V^{\prime} \times T^{\prime}$ for every $\sigma$ in $S$.

2.2.4. $|f(x, t, \sigma)| \leqq c_{1}$ and $\left|f_{x}(x, t, \sigma)\right| \leqq c_{1}$ over $V^{\prime} \times T^{\prime} \times S$.

2.2.5. The set $H(x, t, \alpha)=\left\{(y, \bar{y}) \in E_{n} \times E_{n} \mid y=f(x, t, \sigma), \bar{y}=f_{x}^{T}(x, t, \sigma) \alpha\right.$ for some $\sigma \in S\}$ is compact and convex for every $(x, t, \alpha) \in V^{\prime} \times T^{\prime} \times E_{n}$. (Here $f_{x}^{T}$ is the transpose of the matrix $f_{x}$.)

Remark. Here $V^{\prime}, T^{\prime}$ and $c_{1}$ are as defined in Assumption 2.1. Definition 2.2 differs from the one introduced in [1] and [2] by the addition of Condition 2.2.5.

DefinItion 2.3. Let $B \subset E_{n}$. We shall say that $(C, c(\xi))$ is a proper representation of $B$ at $x$ if

2.3.1. $C$ is a compact and convex set in some euclidean space $E_{k}$.

2.3.2. $c(\xi)$ is a function from $C$ to $B$ which is continuous and continuously differentiable over $C$.

2.3.3. $x=c(\xi)$ for some $\xi \in C$.

In particular, if $B$ is convex, we may choose as $C$ any compact and convex subset of $B$ containing $x$ and we may define $c(\xi)$ as the identity mapping from $C$ to $C$.

There is little difficulty in verifying whether a given $\left(C_{0}, c_{0}\left(\xi_{0}\right)\right)$ resp. $\left(C_{1}, c_{1}\left(\xi_{1}\right)\right)$ is a proper representation of $B_{0}$ resp. $B_{1}$ at some point. It appears much more difficult to determine, in view of condition 2.2.2, whether a given function $f(x, t, \sigma)$ is a proper representation of $F(x, t)$. We indicate, therefore, two methods of constructing such proper representations.

2.4. The Filippov representation. Let $S$ be a compact set in some euclidean space and let $f(x, t, \sigma)$ be continuous over $V^{\prime} \times T^{\prime} \times S$ and satisfy conditions 2.2.1, 2.2.3, 2.2.4 and 2.2.5. Condition 2.2.2 follows from a lemma of Filippov [5, p. 78]. (This lemma and its proof remain valid with our assumptions even though Filippov states slightly stronger assumptions.)

2.5. The Young representation. Let measurable sets over the Hausdorff space $R$ be defined as elements of the smallest Borel field of sets containing open sets of $R$. We then define $S$ as the class of probability measures over $R$ (thus $\sigma \in S$ if $\sigma\left(R_{1}\right)$ is defined for every measurable $R_{1}$ in $R, \sigma$ is completely additive, $\sigma\left(R_{1}\right) \geqq 0$ for every measurable $R_{1}$ in $R$ and $\left.\sigma(R)=1\right)$. We now let

$$
f(x, t, \sigma)=\int_{R} g(x, t, \rho) \sigma(d \rho) .
$$

Conditions 2.2.1, 2.2.2, 2.2.3 and 2.2.4 follow from [1, Theorem 4.1, p. 124]. Condition 2.2 .5 can be easily verified since $R$ is compact and $f(x, t, \sigma)$ is linear in $\sigma$. 
III. Necessary conditions for minimum. A minimax problem. Our principal results can be summarized in

THEOREM 3.1. Let Assumption 2.1 be satisfied. Then there exists a curve $x(t)$ which is a relaxed minimizing curve with respect to $a(x)$ and this curve can be uniformly approximated by solutions of the differential equations (1.1).

Let $f(x, t, \sigma)$ be a proper representation of $F(x, t)$, let $\left(C_{0}, c_{0}\left(\xi_{0}\right)\right)$ resp. $\left(C_{1}, c_{1}\left(\xi_{1}\right)\right)$ be a proper representation of $B_{0}$ at $x\left(t_{0}\right)$ resp. of $B_{1}$ at $x\left(t_{1}\right)$ and let $Z=\{t \in T \mid a(x(t))=0\}$. Let $\delta_{i}=\left(\delta_{i}^{1}, \cdots, \delta_{i}^{n}\right), i=1,2, \cdots, n$, be such that $\delta_{i}^{i}=1$ and $\delta_{j}^{i}=0, i \neq j$. Then either

3.1.1. There exists a point $\xi_{1}^{*}$ in $C_{1}$ such that $c_{1}\left(\xi_{1}^{*}\right)=x\left(t_{1}\right)$ and

$$
c_{1, \xi}^{1}\left(\xi_{1}^{*}\right) \cdot \xi_{1}^{*}=\operatorname{Min}_{\xi_{1} \in C_{1}} c_{1, \xi}^{1}\left(\xi_{1}^{*}\right) \cdot \xi_{1}
$$

(where $c_{1, \xi}^{1}$ is the gradient of $c_{1}^{1}\left(\xi_{1}\right)$, the first component of $c_{1}\left(\xi_{1}\right)$ ), or

3.1.2. There exist a function $\sigma(t)$ from $T$ to $S$, a scalar function $\mu(t)$ over $T$, a function $z(t)$ from $T$ to $E_{n}$, a closed subset $M$ of $Z$, points $\xi_{0}^{*}$ and $\xi_{1}^{*}$ in resp. $C_{0}$ and $C_{1}$ and a non-negative number $\gamma^{1}$ such that

3.1.2.1. $\mu(t) \geqq 0$ and $|z(t)|+\mu(t)>0, t \in T$.

3.1.2.2. $z(t)$ is absolutely continuous on every closed subinterval of $T-M$.

3.1.2.3. $\mu(t)$ is nonincreasing on every subinterval of $T-M, \mu(t)$ is constant on every subinterval of $T-Z$ and $\mu\left(t_{1}\right)=0$ if $t_{1} \in T-Z$.

3.1.2.4.

$$
\begin{aligned}
& \dot{x}(t)=f(x(t), t, \sigma(t)) \text { a.e. in } T, \\
& \dot{z}(t)=-f_{x}^{T}(x(t), t, \sigma(t)) z(t)-\mu(t) b_{x}(x(t), t, \sigma(t)) \text { a.e. in } T-M
\end{aligned}
$$

(where $b(x, t, \sigma)=a_{x}(x) \cdot f(x, t, \sigma)$ and $f_{x}^{T}$ is the transpose of the matrix $f_{x}$ ),

$$
z(t)=o, \quad \mu(t)=1 \quad \text { for } t \in M
$$

and $\lim _{\tau \rightarrow t-0} z(\tau)=z(t-0)=o$ if $t \in M$ and $t$ is the right endpoint of someopen interval of $T-M$, where $o$ is the null-vector of $E_{n}$.

3.1.2.5. (The generalized Weierstrass E-condition.) $\left(z(t)+\mu(t) a_{x}(x(t))\right) \cdot f(x(t), t, \sigma(t))=\underset{\sigma \in S}{\operatorname{Min}}\left(z(t)+\mu(t) a_{x}(x(t))\right) \cdot f(x(t), t, \sigma)$ a.e. in $T$.

3.1.2.6. (Support (transversality) conditions.)

$$
\begin{aligned}
c_{1}\left(\xi_{1}^{*}\right) & =x\left(t_{1}\right), \quad c_{0}\left(\xi_{0}^{*}\right)=x\left(t_{0}\right), \\
\left(\gamma^{1} \delta_{1}-z\left(t_{1}\right)\right) \cdot c_{1, \xi}\left(\xi_{1}^{*}\right) \xi_{1}^{*} & =\operatorname{Min}_{\xi_{1} \in C_{1}}\left(\gamma^{1} \delta_{1}-z\left(t_{1}\right)\right) \cdot c_{1, \xi}\left(\xi_{1}^{*}\right) \xi_{1}, \\
\left(z\left(t_{0}\right)+\mu\left(t_{0}\right) a_{x}\left(x\left(t_{0}\right)\right)\right) \cdot c_{0, \xi}\left(\xi_{0}^{*}\right) \xi_{0}^{*} & =\operatorname{Min}_{\xi_{0} \in C_{0}}\left(z\left(t_{0}\right)+\mu\left(t_{0}\right) a_{x}\left(x\left(t_{0}\right)\right)\right) \cdot c_{0, \xi}\left(\xi_{0}^{*}\right) \xi_{0} .
\end{aligned}
$$

\subsubsection{Either}


3.1.2.7.1. $t_{1} \in Z$ and there exists a non-negative $\gamma^{a}$ such that

or

$$
\left(\gamma^{a} \delta_{1}+a_{x}\left(x\left(t_{1}\right)\right)\right) \cdot c_{1, \xi}\left(\xi_{1}^{*}\right) \xi_{1}^{*}=\underset{\xi_{1} \in C_{1}}{\operatorname{Min}}\left(\gamma^{a} \delta_{1}+a_{x}\left(x\left(t_{1}\right)\right)\right) \cdot c_{1, \xi}\left(\xi_{1}^{*}\right) \xi_{1},
$$

3.1.2.7.2. There exists a point $t_{0}^{*}$ in $T, t_{0}^{*}<t_{1}$, such that

$$
|a(x(t))|+\left|a_{x}(x(t))\right| \neq 0 \text { and }\left|z(t)+\mu(t) a_{x}(x(t))\right| \neq 0 \text { for } t_{0}^{*}<t \leqq t_{1}
$$

and, if $t_{0}^{*}>t_{0}$, then $t_{0}^{*} \in Z$ and either $z\left(t_{0}^{*}\right)=-\gamma a_{x}\left(x\left(t_{0}^{*}\right)\right)$ for some $\gamma \geqq \mu\left(t_{0}^{*}\right)$ or $\left|a_{x}\left(x\left(t_{0}^{*}\right)\right)\right|=\left|a\left(x\left(t_{0}^{*}\right)\right)\right|=0$.

3.1.2.8. If there exists a negative number $\beta$ such that $x \in D$ (where $D$ is as defined in 2.1) and $a(x)=0$ imply $\operatorname{Min}_{\sigma \in S} a_{x}(x) \cdot f(x, t, \sigma) \leqq \beta<0$ for all $t \in T$, then the set $M$ is empty or consists of the single point $t_{0}$.

We shall carry out the proof of this theorem in $\S \S I V$ through VIII. We shall first, however, illustrate the use of the theorem by a very simple example.

3.2. Consider curves $x(t)$ which satisfy conditions (1.1 Relaxed) and (1.2) and, among all such curves, the curve which minimizes $\operatorname{Max}_{t \in T} k(x(t))$, where $k(x)$ is continuous and has continuous first and second order partial derivatives in $V$. This minimax problem can be reduced to finding a relaxed minimizing curve with respect to $a(x)$ in the following manner: let $\boldsymbol{x}=\left(x^{0}, x^{1}, \cdots, x^{n}\right)=\left(x^{0}, x\right), \boldsymbol{g}(x, t, \rho)=\left(0, g^{1}(x, t, \rho), \cdots, g^{n}(x, t, \rho)\right)=(0, g(x, t, \rho))$ and $a(x)=k(x)-x^{0}$.

Let $\boldsymbol{x}(t)=\left(x^{0}(t), x(t)\right)$ be a curve which satisfies the conditions

$$
\begin{aligned}
\dot{x}(t) & =g(x(t), t, \rho(t)) \text { a.e. in } T, \\
x\left(t_{0}\right) & \in B_{0}, \quad x\left(t_{1}\right) \in B_{1}, \\
a(x(t)) & \leqq 0, \quad t \in T,
\end{aligned}
$$

and, among all such curves, minimizes $x^{0}\left(t_{1}\right)$. Then, clearly, $x(t)$ minimizes $x^{0}=\operatorname{Max}_{t \in T} k(x(t))$ among all curves which satisfy (1.1 Relaxed) and (1.2).

We shall now consider, as an illustration, a very simple minimax problem of this kind. Assume that a train is scheduled to cover a unit distance in a unit time and that its acceleration can be varied at will between $-\alpha$ and $\alpha$, where $\alpha>4$. How should the acceleration be varied so that the maximum velocity of the train be minimized?

Let the acceleration of the train at time $t$ be represented by $\sigma(t)$, the velocity by $x^{2}(t)$, the distance covered by $x^{3}(t)$ and let $x^{1}=\operatorname{Max}_{0 \leqq t \leqq 1} x^{2}(t)$. Then we wish to minimize $x^{1}(1)$ subject to the restrictions:

$$
\begin{aligned}
\dot{x}^{1} & =0, \dot{x}^{2}=\sigma, \dot{x}^{3}=x^{2} \text { a.e. in }[0,1], \\
x^{2}(0) & =x^{3}(0)=0, x^{2}(1)=0, x^{3}(1)=1, \\
a(x(t)) & =x^{2}(t)-x^{1}(t) \leqq 0 \text { in }[0,1] .
\end{aligned}
$$


It is clear that the right-hand sides of the differential equations are a proper representation of the convex set $G(x, t)=F(x, t)$. The set $B_{0}$ in $E_{3}$ is the line $x^{2}(0)=x^{3}(0)=0$ and the set $B_{1}$ is the line $x^{2}(1)=0, x^{3}(1)=1$. A proper representation of $B_{i}, i=0,1$, at the point $x(i)$ can be obtained by choosing as $C_{i}$ a compact subinterval of $B_{i}$ in whose relative interior $x(i)$ is contained and choosing as $c_{i}\left(\xi_{i}\right)$ the identity mapping.

We easily verify that Assumption 2.1 holds and Theorem 3.1 is, therefore, applicable. Because $x(1)$ is in the relative interior of $B_{1}$, the alternative 3.1.1 does not hold. We have $\operatorname{Min}_{-\alpha \leqq \sigma \leqq \alpha} a_{x} \cdot f(x, t, \sigma)=\operatorname{Min} \sigma=-\alpha<0$ for all $t \in[0,1]$, hence, by 3.1 .2 .8 , the set $M$ is empty or consists of the single point 0 .

We have, by 3.1.2.4 and 3.1.2.5,

$$
\dot{z}^{1}=0, \dot{z}^{2}=-z^{3}, \dot{z}^{3}=0 \text { a.e. in }[0,1]
$$

and

$$
\sigma(t)=\left\{\begin{aligned}
-\alpha \text { if } v(t) & =z^{2}(t)+\mu(t)>0 \\
\alpha & \text { if } v(t)=z^{2}(t)+\mu(t)<0 \quad \text { a.e. in }[0,1] .
\end{aligned}\right.
$$

Furthermore, by 3.1.2.1, $\mu(t) \geqq 0$ and $\mu(t)+\left|z^{1}\right|+\left|z^{2}(t)\right|+\left|z^{3}\right| \neq 0$ in $[0,1]$. By 3.1.2.6, $z^{1}-\mu(0)=0$.

We easily deduce that $v(t)=z^{2}(0)-z^{3} t+\mu(t)$ and $\mu(0)+\left|z^{2}(0)\right|+\left|z^{3}(0)\right| \neq 0$. Thus the assumption that $\mu(0)=0$ implies, by $(3.1 .2 .3)$, that $\mu(t)=0$ on $[0,1]$ and, by (3.2.5), that

$$
\sigma(t)=\left\{\begin{aligned}
\varepsilon \alpha & \text { a.e. in }[0, \theta], \\
-\varepsilon \alpha & \text { a.e. in }[\theta, 1],
\end{aligned} \text { where } \varepsilon= \pm 1 \text { and } 0 \leqq \theta \leqq 1\right.
$$

This can be easily seen to contradict (3.2.1) and (3.2.2), remembering that $\alpha>4$. Thus $\mu(0)>0$.

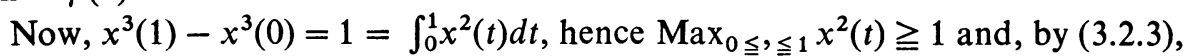
$x^{1} \geqq 1$. Thus $a(x(t))<0$ for all $t$ sufficiently close to 0 and to 1 . Since $\mu(0) \neq 0$, it follows from 3.1.2.3 that $a(x(t))=0$ for some $t \in[0,1]$, hence $Z$ is nonempty. Let $\theta_{1}=\operatorname{Min}_{t \in Z} t$ and $\theta_{2}=\operatorname{Max}_{t \in Z} t$. We have $0<\theta_{1} \leqq \theta_{2}<1$.

If $z^{3} \geqq 0$ then, by 3.1.2.3, v(t) is nonincreasing on [0,1] and constant on $\left[0, \theta_{1}\right)$ and on $\left(\theta_{2}, 1\right]$ and $v\left(\theta_{1}-0\right)>v\left(\theta_{2}+0\right)$. If $v\left(\theta_{2}+0\right) \geqq 0$ then, by (3.2.5), $\sigma(t)=-\alpha$ a.e. in $\left[0, \theta_{1}\right]$, hence $x^{2}\left(\theta_{1}\right)=-\alpha \theta_{1}$, contradicting $x^{2}(t)=x^{1} \geqq 1$ for $t \in Z$. Similarly, $v\left(\theta_{2}+0\right)<0$ implies $\sigma(t)=\alpha$ a.e. in $\left[\theta_{2}, 1\right]$, hence $x^{2}\left(\theta_{2}\right)=-\alpha\left(1-\theta_{2}\right)$, contradicting $\theta_{2} \in Z$. Thus $z^{3}<0$.

Continuing in the same manner, we find that $\theta_{1}<\theta_{2}$, that $v(t)=0$ and $a(x(t))=x^{2}(t)-x^{1}=0$ on $\left[\theta_{1}, \theta_{2}\right]$, which implies $\sigma=0$ a.e. in $\left[\theta_{1}, \theta_{2}\right]$; furthermore, $v(t)<0$ on $\left[0, \theta_{1}\right)$ and $v(t)>0$ on $\left(\theta_{2}, 1\right]$. It follows then easily from (3.2.1), (3.2.2) and (3.2.5) that $\theta_{1}=1 / 2-\sqrt{ }(1 / 4-1 / \alpha), \quad \theta_{2}=1 / 2+\sqrt{ }(1 / 4-1 / \alpha)$, $\sigma(t)=\alpha$ a.e. in $\left[0, \theta_{1}\right], \sigma(t)=0$ a.e. in $\left[\theta_{1}, \theta_{2}\right]$ and $\sigma(t)=-\alpha$ a.e. in $\left[\theta_{2}, 1\right]$. The corresponding value $x^{1}$ of maximum velocity is then $\alpha \theta_{1}$. 
IV. $Q$-minimizing curves.

4.1. It follows from Assumption 2.1 and from [1, Theorem 3.3, p. 123] that there exists a curve $y(t)$ which is a relaxed minimizing curve with respect to $a(x)$.

Let now $f(x, t, \sigma)$ be a proper representation of $F(x, t)$ and let $\left(C_{0}, c_{0}\left(\xi_{0}\right)\right)$ resp. $\left(C_{1}, c_{1}(\xi)\right)$ be a proper representation of $B_{0}$ at $y\left(t_{0}\right)$ resp. of $B_{1}$ at $y\left(t_{1}\right)$.

Consider a finite set $Q=\left\{\tau_{1}, \tau_{2}, \cdots, \tau_{q}\right\}$, where $t_{0}<\tau_{1}<\tau_{2}<\cdots<\tau_{q}<t_{1}$ and the intervals $\left[t_{0}, \tau_{1}\right],\left[\tau_{i}, \tau_{i+1}\right], i=1,2, \cdots, q-1$ and $\left[\tau_{q}, t_{1}\right]$ are all of length not exceeding $\varepsilon_{1} / c_{1}$ (where $\varepsilon_{1}$ and $c_{1}$ are as defined in 2.1). Let $u_{i}(t), i=1, \cdots, q$, be the characteristic function of the interval $\left[t_{0}, \tau_{i}\right]$ in $T$, that is, let $u_{i}(t)=1$ for $t_{0} \leqq t \leqq \tau_{i}$ and $u_{i}(t)=0$ for $\tau_{i}<t \leqq t_{1}, i=1,2, \cdots, q$. Let $\tilde{t}_{0} \in T$, let $\left(\tilde{C}_{0}, \tilde{c}_{0}\left(\tilde{\xi}_{0}\right)\right)$ be a proper representation of some subset $\widetilde{B}$ of $V$ at some point $\tilde{y} \in \widetilde{B}$ and let $\mathscr{I}\left(\tilde{t}_{0}\right)$ be the set of indices $i$ such that $1 \leqq i \leqq q$ and $\tau_{i} \in Q \cap\left(\tilde{t}_{0}, t_{1}\right)$. We shall designate an absolutely continuous curve $x(t), t \in\left[\tilde{t}_{0}, t_{1}\right]$, as a " $Q$-admissible curve $\left(\tilde{t}_{0}, \tilde{C}_{0}, \tilde{c}_{0}\left(\tilde{\xi}_{0}\right)\right)$ "' if there exist points $\tilde{\xi}_{0, Q} \in \tilde{C}_{0}$ and $\xi_{1, Q} \in C_{1}$, a function $\sigma(t)$ from $\left[\tilde{t}_{0}, t_{1}\right]$ to $S$ and absolutely continuous scalar functions $\eta_{i}(t), i \in \mathscr{I}\left(\tilde{t}_{0}\right)$, over $\left[z_{0}, t_{1}\right]$ such that

$$
\begin{aligned}
& \dot{x}(t)=f(x(t), t, \sigma(t)) \text { a.e. in }\left[\tilde{t}_{0}, t_{1}\right] . \\
& \dot{\eta}_{i}(t)=a_{x}(x(t)) \cdot f(x(t), t, \sigma(t)) u_{i}(t)=b(x(t), t, \sigma(t)) u_{i}(t) \text { a.e. in }\left[\tilde{t}_{0}, t_{1}\right], i \in \mathscr{I}\left(\tilde{t}_{0}\right), \\
& x\left(\tilde{t}_{0}\right)=\tilde{c}_{0}\left(\tilde{\xi}_{0, Q}\right), \eta_{i}\left(\tilde{t}_{0}\right)=a\left(\tilde{c}_{0}\left(\tilde{\xi}_{0, Q}\right)\right), i \in \mathscr{I}\left(\tilde{t}_{0}\right), \\
& x\left(t_{1}\right)=c_{1}\left(\xi_{1, Q}\right), \eta_{i}\left(t_{1}\right) \leqq 0, i \in \mathscr{I}\left(\tilde{t}_{0}\right)
\end{aligned}
$$

(where $b(x, t, \sigma)=a_{x}(x) \cdot f(x, t, \sigma)$ and $a_{x}$ is the gradient of $a(x)$ ). We observe that $\eta_{i}\left(t_{1}\right)=a\left(x\left(\tau_{i}\right)\right), i \in \mathscr{I}\left(\tilde{t}_{0}\right)$; hence $a(x(t)) \leqq 0$ for $t \in Q \cap\left(\tilde{t}_{0}, t_{1}\right)$.

A " $Q$-minimizing curve $\left(\tilde{t}_{0}, \tilde{C}_{0}, \tilde{c}_{0}\left(\tilde{\xi}_{0}\right)\right)$ " is a $Q$-admissible curve $\left(\tilde{t}_{0}, \tilde{C}_{0}, \tilde{c}_{0}\left(\tilde{\xi}_{0}\right)\right)$ which minimizes, among all such curves, the value $x^{1}\left(t_{1}\right)$.

We can easily verify (see $2.1 .3,2.2 .1,2.2 .4,2.3 .2$ and 2.3 .3 ) that any $Q$-admissible curve $\left(t_{0}, C_{0}, c_{0}\left(\xi_{0}\right)\right)$ is a relaxed admissible curve with respect to $a(x)-\varepsilon_{1}$ and is, therefore, contained in the compact set $D$ (see 2.1.5). Furthermore, there exists at least one $Q$-admissible curve $\left(t_{0}, C_{0}, c_{0}\left(\xi_{0}\right)\right)$, namely $y(t)$, the previously referred to relaxed minimizing curve with respect to $a(x)$. It follows then, by $\left[1\right.$, Theorem 3.3, p. 123], that there exists a $Q$-minimizing curve $\left(t_{0}, C_{0}, c_{0}\left(\xi_{0}\right)\right)$.

We may now consider a sequence of successively finer sets $Q_{1}, Q_{2}, \cdots$ which become everywhere dense on $T$. Specifically, let $Q_{s}=\left\{t_{0}+k 2^{-s-s_{1}}\left(t_{1}-t_{0}\right) \mid\right.$ $\left.k=1,2, \cdots, 2^{s+s_{1}}-1\right\}, \quad s=1,2, \cdots$, where $s_{1}$ is sufficiently large so that $2^{-s_{1}}\left(t_{1}-t_{0}\right) \leqq \varepsilon_{1} / c_{1}$. As was just shown, for each $s, s=1,2, \cdots$, there exists a curve $x_{s}(t), t \in T$, which is a $Q_{s}$-minimizing curve $\left(t_{0}, C_{0}, c_{0}\left(\xi_{0}\right)\right)$ and each of these curves is contained in the compact set $D$. Then, by [1, Theorems 3.1 and 3.2 , p. 119 and p. 122], 2.1, 2.2 and 2.3, there exists an infinite sequence $P$ of integers, an absolutely continuous curve $x^{*}(t), t \in T$, and a function $\sigma(t)$ from $T$ 
to $S$ such that the curves $x_{s}(t), s$ in $P$, converge uniformly to $x^{*}(t)$ over $T$ and furthermore,

$$
\begin{aligned}
\dot{x}^{*}(t) & =f\left(x^{*}(t), t, \sigma(t)\right) \quad \text { a.e. in } T, \\
x^{*}(t) & \in D, t \in T, \\
x^{*}\left(t_{0}\right) & =c_{0}\left(\xi_{0}\right), x^{*}\left(t_{1}\right)=c_{1}\left(\xi_{1}\right) \text { for some } \xi_{0} \in C_{0} \text { and some } \xi_{1} \in C_{1} .
\end{aligned}
$$

We have

LEMMA 4.2. The curves $x_{s}(t), s$ in P, satisfy a uniform Lipschitz condition over $T$ and the curve $x^{*}(t)$ is a relaxed minimizing curve with respect to $a(x)$.

Proof. The first part of the lemma follows directly from (4.1.1) and 2.2.4. Since the sets $Q_{s}$ become everywhere dense on $T$, it easily follows that $a\left(x^{*}(t)\right) \leqq 0$ over $T$. Thus, by (4.1.2), $x^{*}(t)$ is a relaxed admissible curve with respect to $a(x)$. Furthermore, as previously observed, the curve $y(t)$ (which is a relaxed minimizing curve with respect to $a(x))$ is also a $Q_{s}$-admissible curve $\left(t_{0}, C_{0}, c_{0}\left(\xi_{0}\right)\right)$ for every $s$, hence $x_{s}^{1}\left(t_{1}\right) \leqq y^{1}\left(t_{1}\right)$ implying $x^{* 1}\left(t_{1}\right) \leqq y^{1}\left(t_{1}\right)$. This proves that $x^{*}(t)$ is a relaxed minimizing curve with respect to $a(x)$.

We now investigate certain properties of $Q$-minimizing curves.

LEMMA 4.3. Let $x_{s}(t)$ be a $Q_{s}$-minimizing curve $\left(t_{0}, C_{0}, c_{0}(\xi)\right)$ for any fixed $s$ and let $Z_{s}=\left\{t \in Q_{s} \mid a\left(x_{s}(t)\right)=0\right\}$. Then either there exists a point $\xi_{1, s}$ in $C_{1}$ such that

$$
c_{1}\left(\xi_{1, s}\right)=x_{s}\left(t_{1}\right) \text { and } c_{1, \xi}^{1}\left(\xi_{1, s}\right) \xi_{1, s}=\operatorname{Min}_{\xi_{1} \in C_{1}} c_{1, \xi}^{1}\left(\xi_{1, s}\right) \xi_{1}
$$

(where $c_{1, \xi}=\left(c_{1, \xi}^{1}, \cdots, c_{1, \xi}^{n}\right)$ and $c_{1, \xi}^{i}$ is the gradient of $\left.c_{1}^{i}\left(\xi_{1}\right), i=1, \cdots, n\right)$ or there exist a non-negative number $\gamma_{s}^{1}$, points $\xi_{0, s}$ in $C_{0}$ and $\xi_{1, s}$ in $C_{1}$, a function $\sigma_{s}(t)$ from $T$ to $S$, a set $L_{s} \subset Z_{s}$ and functions $z_{s}(t), w_{s}(t)$ and $\mu_{s}(t)$ from $T$ to resp. $E_{n}, E_{1}$ and $E_{1}$ such that

4.3.1. $x_{s}\left(t_{0}\right)=c_{0}\left(\xi_{0, s}\right)$ and $x_{s}\left(t_{1}\right)=c_{1}\left(\xi_{1, s}\right)$.

4.3.2. $\mu_{s}(t) \geqq 0$ and $\left|z_{s}(t)\right|+\mu_{s}(t)>0$ for $t \in T$.

4.3.3. $z_{s}(t)$ is absolutely continuous on any closed subinterval of $T-L_{s}$ and $z_{s}(t)$ is continuous from the left over $T$.

4.3.4. $\mu_{s}\left(t_{1}\right)=0$ and $\mu_{s}(t)$ is, on every subinterval of $T-L_{s}$, a nonincreasing step function, continuous from the left, with no discontinuities except possibly at points of $Z_{s}$.

4.3.5. $w_{s}(t)=1$ for $t \in L_{s}$, where $w_{s}(t)=\mu_{s}(t) /\left(\left|z_{s}(t)\right|+\mu_{s}(t)\right), t \in T$.

4.3.6. $\dot{x}_{s}(t)=f\left(x_{s}(t), t, \sigma_{s}(t)\right)$ a.e. in $T ; \quad \dot{z}_{s}(t)=-f_{x}^{T}\left(x_{s}(t), t, \sigma_{s}(t)\right) z_{s}(t)$ $-\mu_{s}(t) b_{x}\left(x_{s}(t), t, \sigma_{s}(t)\right)$ a.e. in $T$, where $f_{x}^{T}$ is the transpose of the matrix $f_{x}$ and $b(x, t, \sigma)=a_{x}(x) \cdot f(x, t, \sigma)$.

4.3.7. $v_{s}(t) \cdot f\left(x_{s}(t), t, \sigma_{s}(t)\right)=\operatorname{Min}_{\sigma \in S} v_{s}(t) \cdot f\left(x_{s}(t), t, \sigma\right)$ a.e. in $T$, where $v_{s}(t)=z_{s}(t)+\mu_{s}(t) a_{x}\left(x_{s}(t)\right), t \in T$. 
4.3.8. $v_{s}\left(t_{0}\right) \cdot c_{0, \xi}\left(\xi_{0, s}\right) \xi_{0, s}=\operatorname{Min}_{\xi_{0} \in c_{0}} v_{s}\left(t_{0}\right) \cdot c_{0, \xi}\left(\xi_{0, s}\right) \xi_{0}$.

4.3.9. $\left(\gamma_{s}^{1} \delta_{1}-z_{s}\left(t_{1}\right)\right) \cdot c_{1, \xi}\left(\xi_{1, s}\right) \xi_{1, s}=\operatorname{Min}_{\xi_{1} \in C_{1}}\left(\gamma_{s}^{1} \delta_{1}-z_{s}\left(t_{1}\right)\right) \cdot c_{1, \xi}\left(\xi_{1, s}\right) \xi_{1}$, where $\delta_{1}=\left(\delta_{1}^{1}, \cdots, \delta_{1}^{n}\right), \delta_{1}^{1}=1$ and $\delta_{1}^{j}=0, j=2, \cdots, n$.

Proof. Let us write $x(t), \sigma(t), z(t), \mu(t), v(t), w(t)$ instead of $x_{s}(t), \cdots, w_{s}(t)$. We have shown, in 4.1 , that $x(t)$, a $Q_{s}$-minimizing curve $\left(t_{0}, C_{0}, c_{0}\left(\xi_{0}\right)\right)$ exists and is contained in the compact set $D$. It follows then easily from relations (4.1.1) that $\eta_{i}(t), i=1,2, \cdots, q, t \in T, q=2^{s_{1}+s}-1$, also exist and $\eta_{i}(t)=a(x(t))$ over $\left[t_{0}, \tau_{i}\right]$ and $\eta_{i}(t)=a\left(x\left(\tau_{i}\right)\right)$ over $\left[\tau_{i}, t_{1}\right]$. Thus the curve $\left(x(t), \eta_{1}(t), \cdots, \eta_{q}(t)\right)$ is a relaxed minimizing curve in the sense of [2, Theorem 6.1, p. 142] (where $V=V^{\prime} \times V_{\eta}$, $A=D \times D_{\eta}, V_{\eta}=I_{a} \times I_{a} \times \cdots \times I_{a}(q$ times $), D_{\eta}=R_{a} \times R_{a} \times \cdots \times R_{a}(q$ times $), R_{a}$ is the range of $a(x)$ for $x \in D$ and $I_{a}$ is a bounded open interval containing $R_{a}$ ) for the problem defined by relations (4.1.1). It follows then, after some manipulation, from $[2$, Theorem $6.1, \mathrm{p} .142]\left({ }^{3}\right)$ that either there exists a point $\xi_{1, s}$ in $C_{1}$ such that

4.3.10. $\quad c_{1}\left(\xi_{1, s}\right)=x\left(t_{1}\right)$ and $c_{1, \xi}^{1}\left(\xi_{1, s}\right) \cdot \xi_{1, s}=\operatorname{Min}_{\xi_{1} \in C_{1}} c_{1, \xi}^{1}\left(\xi_{1, s}\right) \cdot \xi_{1}$, or there exist a non-negative number $\gamma_{s}^{1}$, points $\xi_{0, s}$ in $C_{0}$ and $\xi_{1, s}$ in $C_{1}$ and absolutely continuous functions $z(t), v_{1}(t), \cdots, v_{q}(t)$ from $T$ to resp. $E_{n}, E_{1}, \cdots, E_{1}$ such that

4.3.11. $|z(t)|+\sum_{i=1}^{q}\left|v_{1}(t)\right| \neq 0, t \in T$;

$$
\dot{x}(t)=f(x(t), t, \sigma(t)) \text { a.e. in } T \text {, }
$$

4.3.12. $\dot{z}(t)=-f_{x}^{T}(x(t), t, \sigma(t)) z(t)-\sum_{i=1}^{q} v_{i}(t) u_{i}(t) b_{x}(x(t), t, \sigma(t))$ a.e. in $T$,

$$
\dot{v}_{i}(t)=0, i=1,2, \cdots, q \text { a.e. in } T \text {; }
$$

4.3.13. $v(t) \cdot f(x(t), t, \sigma(t))=\operatorname{Min}_{\sigma \in S} v(t) \cdot f(x(t), t, \sigma)$ a.e. in $T$, where $v(t)=z(t)$ $+\sum_{i=1}^{q} v_{i}(t) u_{i}(t) \cdot a_{x}(x(t))$

4.3.14. $\quad c_{0}\left(\xi_{0, s}\right)=x\left(t_{0}\right)$ and $v\left(t_{0}\right) \cdot c_{0, \xi}\left(\xi_{0, s}\right) \xi_{0, s}=\operatorname{Min}_{\xi_{0} \in c_{0}} v\left(t_{0}\right) \cdot c_{0, \xi}\left(\xi_{0, s}\right) \xi_{0}$.

4.3.15. $\quad c_{1}\left(\xi_{1, s}\right)=x\left(t_{1}\right),\left(\gamma_{s}^{1} \delta_{1}-z\left(t_{1}\right)\right) \cdot c_{1, \xi}\left(\xi_{1, s}\right) \xi_{1, s}=\operatorname{Min}_{\xi_{1} \in c_{1}}\left(\gamma_{s}^{1} \delta_{1}-z\left(t_{1}\right)\right)$ $\cdot c_{1, \xi}\left(\xi_{1, s}\right) \xi_{1}, \quad v_{i}\left(t_{1}\right) \geqq 0, \quad i=1,2, \cdots, q$ and $v_{i}\left(t_{1}\right)=0$ if $\eta_{i}\left(t_{1}\right)=a\left(x\left(\tau_{i}\right)\right)<0$, $i=1, \cdots, q$.

Assume that 4.3.10 does not hold.

Let $\mu(t)=\sum_{i=1}^{q} v_{i}(t) u_{i}(t)$ and let $\tau_{0}=t_{0}, \tau_{q+1}=t_{1}$. We observe that, by 4.3.12 and 4.3.15, $v_{i}(t)=v_{i}$ is constant and $v_{i} \geqq 0, i=1,2, \cdots, q$, and thus $\mu(t)$ is a non-negative nonincreasing step function, continuous from the left, with its discontinuities, if any, restricted to points of $Z_{s}$. Furthermore, $\mu\left(t_{1}\right)=0$.

We shall now show that there exists an integer $j, 1 \leqq j \leqq q+1$, such that $|z(t)|+\mu(t)>0$ over $\left[t_{0}, \tau_{j}\right]$. Indeed, if $\mu\left(\tau_{j}\right)>0$ for some $j, 1 \leqq j \leqq q+1$, then $\mu(t) \geqq \mu\left(\tau_{j}\right)>0$ for all $t$ in $\left[t_{0}, \tau_{j}\right]$. If $\mu\left(\tau_{j}\right)=0$ for all $j, 1 \leqq j \leqq q+1$, then $v_{j}=0,1 \leqq j \leqq q$, hence, by 4.3.11, $|z(t)| \neq 0, t \in T$, and $|z(t)|+\mu(t)>0$ over $\left[t_{0}, t_{1}\right]$.

(3) The last (third) line of (6.1.3) in [2, p. 143] should read "for some $\zeta^{1} \geqq 0$ " instead of "for some $\xi^{1} \geqq 0$." 
Let now $\theta_{1}$ be the largest of the numbers $\tau_{j}, j=1, \cdots, q+1$, such that $|z(t)|+\mu(t)>0$ over $\left[t_{0}, \tau_{j}\right]$. If $\theta_{1}=t_{1}$, we define $L_{s}$ to be the empty set and the lemma follows easily from relations 4.3.11 through 4.3.15. If $\theta_{1}=\tau_{j}<t_{1}$ then $\left|z\left(\tau_{j+1}\right)\right|=0$ and $\mu\left(\tau_{j+1}\right)=0$ implying that $\mu(t)=0$ over $\left[\tau_{j+1}, t_{1}\right]$. It follows then from 4.3.12 that $|z(t)|=0$ over $\left[\theta_{1}, t_{1}\right]$ and

$$
w\left(\theta_{1}\right)=\mu\left(\theta_{1}\right) /\left(\left|z\left(\theta_{1}\right)\right|+\mu\left(\theta_{1}\right)\right)=1 .
$$

Furthermore, $v_{j} \neq 0$; hence, by $4.3 .15, a\left(x\left(\theta_{1}\right)\right)=0$ and $\theta_{1} \in Z_{s}$.

We observe that, trivially, $x(t)$ is a $Q^{\prime}$-minimizing curve $\left(\theta_{1},\left\{x\left(\theta_{1}\right)\right\}\right.$, identity) where $Q^{\prime}=Q_{s} \cap\left(\theta_{1}, t_{1}\right),\left\{x\left(\theta_{1}\right)\right\}$ is the set with the single element $x\left(\theta_{1}\right)$ and the mapping is the identity mapping of $\left\{x\left(\theta_{1}\right)\right\}$ into itself. It follows now, by our previous argument, that there exists a point $\theta_{1}^{\prime}$ in $Z_{s} \cap\left(\theta_{1}, t_{1}\right] \cup\left\{t_{1}\right\}$, functions $z^{\prime}(t)$, $\mu^{\prime}(t)$ and $w^{\prime}(t)$ over $\left[\theta_{1}, \theta_{1}^{\prime}\right]$ and a non-negative number $\bar{\gamma}_{s}^{1}$ such that $\mu^{\prime}(t)$ is a non-negative nonincreasing step function over $\left[\theta_{1}, \theta_{1}^{\prime}\right]$, continuous from the left, and with its only possible discontinuities on $\left(\theta_{1}, \theta_{1}^{\prime}\right] \cap Z_{s}, z^{\prime}(t)$ is absolutely continuous on $\left[\theta_{1}, \theta_{1}^{\prime}\right],\left|z^{\prime}(t)\right|+\mu^{\prime}(t)>0$ on $\left(\theta_{1}, \theta_{1}^{\prime}\right], w^{\prime}\left(\theta_{1}^{\prime}\right)=\mu^{\prime}\left(\theta_{1}^{\prime}\right) /\left(\left|z^{\prime}\left(\theta_{1}^{\prime}\right)\right|+\mu^{\prime}\left(\theta_{1}^{\prime}\right)\right)=1$ if $\theta_{1}^{\prime}<t_{1}$ and relations (4.3.6), (4.3.7) and (4.3.9) are satisfied with $\left[\theta_{1}, \theta_{1}^{\prime}\right]$ replacing $T$.

Continuing in this manner if $\theta_{1}^{\prime}<t_{1}$, after at most $q+1$ steps we shall determine a point $\theta_{1}{ }^{\prime} \cdots^{\prime}=t_{1}$, since $\theta_{1}{ }^{\prime} \cdots^{\prime} \in Q_{s}$ and $\theta_{1}{ }^{\prime} \cdots^{\prime}<\theta_{1}{ }^{\prime} \cdots{ }^{\prime \prime}$. We now redefine the functions $z(t)$ and $\mu(t)$ to equal resp. $z^{\prime} \ldots{ }^{\prime \prime}(t)$ and $\mu^{\prime} \ldots{ }^{\prime \prime}(t)$ over $\left(\theta_{1}{ }^{\prime} \cdots^{\prime}, \theta_{1}{ }^{\prime} \cdots{ }^{\prime \prime}\right]$, we let $\gamma_{s}^{1}$ be the number $\bar{\gamma}_{s}^{1}$ obtained in the last step and we define $L_{s}$ to be the set of the points $\theta_{1}, \theta_{1}^{\prime}, \cdots$ which are less than $t_{1}$. The lemma now follows directly.

V. Certain properties of $z_{s}(t)$ and $w_{s}(t)$.

5.1. We shall continue to use the notation introduced in the previous section. If, for infinitely many values of $s$ in $P$, there exists a point $\xi_{1, s}$ in $C_{1}$ such that

$$
c_{1}\left(\xi_{1, s}\right)=x_{s}\left(t_{1}\right) \text { and } c_{1, \xi}^{1}\left(\xi_{1, s}\right) \xi_{1, s}=\operatorname{Min}_{\xi_{1} \in C_{1}} c_{1, \xi}^{1}\left(\xi_{1, s}\right) \xi_{1}
$$

then, $C_{1}$ being compact, we may extract an infinite subsequence $P^{\prime}$ of $P$ such that $\xi_{1, s}$ converges to some $\xi_{1}^{*}$ over $P^{\prime}$ and we have

$$
c_{1}\left(\xi_{1}^{*}\right)=x^{*}\left(t_{1}\right) \text { and } c_{1, \xi}^{1}\left(\xi_{1}^{*}\right) \xi_{1}^{*}=\operatorname{Min}_{\xi_{1} \in C_{1}} c_{1, \xi}^{1}\left(\xi_{1}^{*}\right) \xi_{1} .
$$

The alternative 3.1.1 of Theorem 3.1 is then satisfied.

We shall henceforth assume, therefore (unless otherwise specified), that the second alternative of Lemma 4.3 holds for all sufficiently large values of $s$ in $P$ which constitute a sequence $P_{1}$ of integers.

Lemma 5.2. Let $s$ be in $P_{1}$ and let $t$ and $t^{\prime}$ belong to a subinterval of $T-L_{s}$. Then there exists a positive constant $c_{2}$ depending only on $a(x), t_{1}-t_{0}$ and $c_{1}$ and $D$ (of Assumption 2.1) and such that 
5.2.1. $z_{s}(t)=z_{s}\left(t^{\prime}\right)+\left(\left|z_{s}\left(t^{\prime}\right)\right|+\mu_{s}\left(\operatorname{Min}\left(t, t^{\prime}\right)\right)\right) \alpha_{s}\left(t, t^{\prime}\right)$, where $\left|\alpha_{s}\left(t, t^{\prime}\right)\right|$ $\leqq c_{2} \cdot\left|t-t^{\prime}\right|$ and

5.2.2. $\left|z_{s}(t)\right| \leqq c_{2}\left|z_{s}\left(t^{\prime}\right)\right|+c_{2}\left|\int_{t}^{t^{\prime}} \mu_{s}(\tau) d \tau\right|$.

Proof. As was observed in 4.1, the curve $x_{s}(t)$ is contained in the compact set $D$ (of Assumption 2.1). Since $a(x)$ has continuous first and second order partial derivatives over $V^{\prime}$, and since, by 2.2.4, $|f(x, t, \sigma)| \leqq c_{1}$ and $\left|f_{x}(x, t, \sigma)\right| \leqq c_{1}$ over $V^{\prime} \times T^{\prime} \times S$, it follows that there exists a constant $c_{2}^{\prime}$ such that $\left|b\left(x_{s}(t), t, \sigma_{s}(t)\right)\right| \leqq c_{2}^{\prime}$ and $\left|b_{x}\left(x_{s}(t), t, \sigma_{s}(t)\right)\right| \leqq c_{2}^{\prime}$ a.e. in $T$.

It can be easily verified that the second equation of 4.3.6 yields

$$
z_{s}(t)=U_{s}^{T}\left(t, t^{\prime}\right) z_{s}\left(t^{\prime}\right)+\int_{t}^{t^{\prime}} U_{s}^{T}(t, \tau) b_{x}\left(x_{s}(\tau), \tau, \sigma_{s}(\tau)\right) \mu_{s}(\tau) d \tau,
$$

where the matrix $U_{s}(t, \tau)$ is the solution of the system

$$
\frac{d}{d \tau} U_{s}(t, \tau)=f_{s}\left(x_{s}(\tau), \tau, \sigma_{s}(\tau)\right) U_{s}(t, \tau)
$$

which reduces to the unit matrix $I$ for $\tau=t$. It follows easily that there exists a constant $c_{2}=c_{2}\left(c_{1}, c_{2}^{\prime}\right)$ such that $\left|U_{s}\left(t, t^{\prime}\right)\right| \leqq c_{2},\left|U_{s}^{T}(t, \tau) b_{x}\left(x_{s}(\tau), \tau, \sigma_{s}(\tau)\right)\right| \leqq c_{2}$, $\left|(t-\tau)^{-1}\left(U_{s}^{T}(t, \tau)-I\right)\right| \leqq c_{2}$ and $\left|f_{s}\left(x_{s}(\tau), \tau, \sigma_{s}(\tau)\right)\right| \leqq c_{2}$.

Relations 5.2.1 and 5.2.2 now follow from the fact that $\mu_{s}(\tau) \geqq 0$ and $\mu_{s}(\tau)$ is nonincreasing between $t$ and $t^{\prime}$.

Lemma 5.3. Let $s$ be in $P_{1}$. Then $w_{s}(t)-c_{2} t$ is a nonincreasing function over $T$.

Proof. Let $t<t^{\prime}$ and $\left[t, t^{\prime}\right) \subset T-L_{s}$. Then $\mu_{s}(t) \geqq \mu_{s}\left(t^{\prime}\right) \geqq 0$ and, by 5.2.1 and 4.3.4,

$$
\left|z_{s}(t)\right|+\mu_{s}(t) \leqq\left(\left|z_{s}\left(t^{\prime}\right)\right|+\mu_{s}(t)\right)(1+\alpha), \text { where } 0 \leqq \alpha \leqq c_{2}\left(t^{\prime}-t\right) .
$$

Now

$$
\begin{aligned}
w_{s}(t) & =\frac{\mu_{s}(t)}{\left|z_{s}(t)\right|+\mu_{s}(t)} \geqq \frac{\mu_{s}(t)}{(1+\alpha)\left(\left|z_{s}\left(t^{\prime}\right)\right|+\mu_{s}(t)\right)} \\
& \geqq \frac{\mu_{s}\left(t^{\prime}\right)}{(1+\alpha)\left(\left|z_{s}\left(t^{\prime}\right)\right|+\mu_{s}\left(t^{\prime}\right)\right)} \geqq w_{s}\left(t^{\prime}\right)(1-\alpha) \geqq w_{s}\left(t^{\prime}\right)-c_{2}\left(t^{\prime}-t\right) .
\end{aligned}
$$

Let now $t<t^{\prime}$ and let $t$ and $t^{\prime}$ be arbitrary points of $T$. We have just proven the lemma for the case when $\left[t, t^{\prime}\right) \subset T-L_{s}$. If $t \in L_{s}$, then by $4.3 .5, w_{s}(t)=1$ and the lemma is trivially satisfied. In all other cases, let $\theta$ be the point in $\left[t, t^{\prime}\right] \cap L_{s}$ nearest $t$. Then, by $4.3 .5, w_{s}(\theta)=1$ and, by (5.3.1),

$$
w_{s}(t) \geqq 1-c_{2}(\theta-t) \geqq w_{s}\left(t^{\prime}\right)-c_{2}\left(t^{\prime}-t\right) .
$$




\section{Passing to the limit.}

6.1. Let us consider, for all $s$ in the infinite sequence $P_{1}$, the points $\xi_{0, s}$ and $\xi_{1, s}$ and the functions $w_{s}(t)$. Since $\xi_{0, s} \in C_{0}$ and $\xi_{1, s} \in C_{1}$ and since $C_{0}$ and $C_{1}$ are compact, there exists an infinite subsequence $P_{1}^{\prime}$ of $P_{1}$ and points $\xi_{0}^{*} \in C_{0}$ and $\xi_{1}^{*} \in C_{1}$ such that $\xi_{0, s}$ converges to $\xi_{0}^{*}$ and $\xi_{1, s}$ converges to $\xi_{1}^{*}$ over the sequence $P_{1}^{\prime}$. Furthermore, $0 \leqq w_{s}(t) \leqq 1$ for $s$ in $P_{1}^{\prime}$ and $t \in T$, and, by Lemma 5.3, $w_{s}(t)$ are of uniformly bounded variation over $T$ for all $s$ in $P_{1}^{\prime}$. It follows thus, by Helly's selection theorem, that there exists an infinite subsequence $P_{2}$ of $P_{1}^{\prime}$ such that $w_{s}(t)$ converges to a function $w^{*}(t)$ over $T$ as $s \rightarrow \infty$ over $P_{2}$. In view of Lemma 5.3, the function $w^{*}(t)-c_{2} t$ is nonincreasing over $T$.

Let now $Z=\left\{t \in T \mid a\left(x^{*}(t)\right)=0\right\}$ and let $L=\left\{t \in T \mid \lim \sup _{\tau \rightarrow} w_{t}{ }^{*}(\tau)=1\right.$ or $\left.w^{*}(t)=1\right\}$. Clearly $Z$ and $L$ are closed sets and $T-L \cap Z$ is open (relative to $T$ ). We shall say that " $J$ is a maximal subinterval of $T-L \cap Z$ " or, briefly, that " $J$ is maximal" if $J \subset T-L \cap Z, J$ is convex and open relative to $T$, and the relation $J \subset K \subset T-L \cap Z$ implies $J=K$ for every interval (convex set) $K$.

We let $\tau_{0}(J), \tau_{1}(J)$ and $\tau^{*}(J)$ represent respectively the left and the right endpoints and the midpoint of $J$. Thus, if $J$ is maximal, $\tau_{0}(J) \in J$ if and only if $\tau_{0}(J)=t_{0}$ and $t_{0} \in T-L \cap Z$; and $\tau_{1}(J) \in J$ if and only if $\tau_{1}(J)=t_{1}$ and $t_{1} \in T-L \cap Z$. Furthermore, for every maximal $J$ and for every $s$ in $P_{2}$, we set

$$
z_{s}^{*}(t)=\frac{z_{s}(t)}{\left|z_{s}\left(\tau^{*}(J)\right)\right|+\mu_{s}\left(\tau^{*}(J)\right)}, \quad t \in J,
$$

$$
\begin{aligned}
& \mu_{s}^{*}(t)=\frac{\mu_{s}(t)}{\left|z_{s}\left(\tau^{*}(J)\right)\right|+\mu_{s}\left(\tau^{*}(J)\right)}, \quad t \in J, \\
& v_{s}^{*}(t)=z_{s}^{*}(t)+\mu_{s}^{*}(t) a_{x}\left(x_{s}(t)\right)=\frac{v_{s}(t)}{\left|z_{s}\left(\tau^{*}(J)\right)\right|+\mu_{s}\left(\tau^{*}(J)\right)}, \quad t \in J .
\end{aligned}
$$

These definitions are permissible since, by 4.3 .2 , the denominator is positive. We also observe that, by 4.3.6,

$$
\dot{z}_{s}^{*}(t)=-f_{x}^{T}\left(x_{s}(t), t, \sigma_{s}(t)\right) z_{s}^{*}(t)-\mu_{s}^{*}(t) b_{x}\left(x_{s}(t), t, \sigma_{s}(t)\right) \text { a.e. in } T-L \cap Z .
$$

LEMMA 6.2. Let $J$ be maximal. Given any closed subinterval $\left[\theta_{0}, \theta_{1}\right]$ of $J$, the sets $L_{s} \cap\left[\theta_{0}, \theta_{1}\right]$ are empty and the functions $z_{s}^{*}(t)$ are absolutely continuous on that subinterval for sufficiently large $s$ in $P_{2}$.

Proof. Assume that $L_{s} \cap\left[\theta_{0}, \theta_{1}\right]$ are nonempty for infinitely many values of $s$ in $P_{2}$. Then $\left[\theta_{0}, \theta_{1}\right]$ contains points $l_{s}$ in $L_{s}$ for all $s$ belonging to an infinite subsequence $P_{2}^{\prime}$ of $P_{2}$. By 4.3.5, $w_{s}\left(l_{s}\right)=1$ for all $s$ in $P_{2}^{\prime}$. Let $\theta$ be the limit of some convergent subsequence of such $l_{s}$ and let $\eta=0$ if $\theta=\theta_{0}$ and let otherwise $\eta>0$ and $\theta-\eta \in\left[\theta_{0}, \theta_{1}\right]$. Then, by Lemma 5.3, $w_{s}(\theta-\eta)-c_{2}(\theta-\eta)$ $\geqq w_{s}\left(l_{s}\right)-c_{2} l_{s}=1-c_{2} l_{s}$ for all $s$ in $P_{2}^{\prime}$ such that $\theta-\eta \leqq l_{s}$. It follows that $w^{*}(\theta-\eta) \geqq 1-c_{2} \eta$, implying that $\theta \in L$. 
Now, $l_{s} \in L_{s} \subset Z_{s}$ and $a\left(x_{s}\left(l_{s}\right)\right)=0, s$ in $P_{2}^{\prime}$, implying, by Lemma 4.2 , that $a\left(x^{*}(\theta)\right)=0$ and $\theta \in Z$. Thus $\theta \in L \cap Z$ contradicting $\theta \in\left[\theta_{0}, \theta_{1}\right] \subset J \subset T-L \cap Z$.

It follows now from 4.3.3 that $z_{s}(t)$, hence also $z_{s}^{*}(t)$, are absolutely continuous on $\left[\theta_{0}, \theta_{1}\right]$ for sufficiently large $s$.

LEMMA 6.3. For every fixed $t$ in $T-L \cap Z$, the functions $\mu_{s}^{*}(t)$ and $z_{s}^{*}(t)$ are bounded over $P_{2}$. Furthermore,

$$
\left|z_{s}^{*}(t)\right| \leqq c_{2}+c_{2}\left(t_{1}-t_{0}\right)\left(\mu_{s}^{*}(t)+1\right) \text {, s in } P_{2}, t \in T-L \cap Z .
$$

Proof. Assume, by way of contradiction, that there exists a point $t$ in $T-L \cap Z$ such that $\mu_{s}^{*}(t)$ is unbounded over $P_{2}$. Let $J$ be the maximal subinterval of $T-L \cap Z$ to which $t$ belongs and let $\tau^{*}=\tau^{*}(J)$. By Lemma 6.2, the closed interval joining $t$ and $\tau^{*}$ contains no points of $L_{s}$ for sufficiently large $s$ in $P_{2}$, say for $s$ in $P_{2}^{\prime}$. Thus, by $4.3 .4, \mu_{s}(t)$ and $\mu_{s}^{*}(t)$ are non-negative and nonincreasing on that closed interval for all $s$ in $P_{2}^{\prime}$. Since $\mu_{s}^{*}\left(\tau^{*}\right) \leqq 1$ for all $s$ in $P_{2}$, it follows that $t<\tau^{*}$.

Let now $U$ be the set of all such points $t$ belonging to $J$ and let $\theta$ be the least upper bound of $U$. Clearly $\theta \leqq \tau^{*}$ and $\theta \in J$. We have

$$
\theta \in Z \text {. }
$$

Otherwise, for some positive $\delta, \delta<\tau_{1}(J)-\theta$, and for all sufficiently large $s$ in $P_{2}$, we have $a\left(x_{s}(t)\right) \neq 0$ over $J \cap[\theta-\delta, \theta+\delta]$, implying, by 4.3 .4 , that $\mu_{s}(t)$ and $\mu_{s}^{*}(t)$ are constant over $J \cap[\theta-\delta, \theta+\delta]$. Since $[\theta-\delta, \theta+\delta]$ contains points of $U$ and $[\theta, \theta+\delta] \subset J$, this contradicts the definition of $\theta$ as an upper bound of $U$.

By the definition of $U$ and of $\theta, \psi(t)=\lim \sup _{P_{2}} \mu_{s}^{*}(t)$ exists for every $t>\theta, t \in J$ and, by 4.3 .4 and Lemma 6.2 , it is nonincreasing on $\left(\theta, \tau^{*}\right]$. Let now $0<\eta<\tau_{1}(J)-\theta, \theta^{\prime \prime}=\operatorname{Min}\left(\tau^{*}, \theta+\eta\right)$ and let $\theta^{\prime}$ be a point in $U$ such that $\theta-\theta^{\prime} \leqq \eta$. Then, by Lemma $6.2, \mu_{s}^{*}(t)$ is nonincreasing on $\left[\theta^{\prime}, \tau^{*}\right]$ for sufficiently large $s$ in $P_{2}$ and liminf $P_{P_{2}}\left(\mu_{s}^{*}\left(\theta^{\prime}\right)\right)^{-1} \int_{\theta^{\prime}}^{\tau^{*}} \mu_{s}^{*}(\tau) d \tau \leqq \liminf _{P_{2}}\left(\mu_{s}^{*}\left(\theta^{\prime}\right)\right)^{-1} \int_{\theta^{\prime \prime}}^{\tau^{*}} \psi(\tau) d \tau+\int_{\theta^{\prime}}^{\theta^{\prime \prime}} d \tau$.

Since $\mu_{s}^{*}\left(\theta^{\prime}\right)$ is unbounded (because $\theta^{\prime} \in U$ ), it follows that

$$
\underset{P_{2}}{\liminf }\left(\mu_{s}^{*}\left(\theta^{\prime}\right)\right)^{-1} \int_{\theta^{\prime}}^{\tau^{*}} \mu_{s}^{*}(\tau) d \tau \leqq \theta^{\prime \prime}-\theta^{\prime} \leqq 2 \eta
$$

hence, by Lemmas 5.2 and 6.2 (setting $t=\theta^{\prime}$ and $t^{\prime}=\tau^{*}$ in 5.2.2),

$$
\underset{\boldsymbol{P}_{2}}{\liminf }\left|z_{s}^{*}\left(\theta^{\prime}\right)\right| / \mu_{s}^{*}\left(\theta^{\prime}\right) \leqq 2 c_{2} \eta \text {. }
$$

This last inequality implies that

$$
w^{*}\left(\theta^{\prime}\right)=\lim _{P_{2}} w_{s}\left(\theta^{\prime}\right)=\lim _{P_{2}} \frac{\mu_{s}^{*}\left(\theta^{\prime}\right)}{\left|z_{s}^{*}\left(\theta^{\prime}\right)\right|+\mu_{s}^{*}\left(\theta^{\prime}\right)} \geqq \frac{1}{1+2 c_{2} \eta} \geqq 1-2 c_{2} \eta .
$$


Since $\eta$ may be chosen arbitrarily small, it follows that $\theta \in L$, contradicting, in view of (6.3.1), our previous conclusion that $\theta \in J$.

Thus $\mu_{s}^{*}(t), s$ in $P_{2}$, are bounded for every fixed $t$ in $J$. By Lemmas 5.1 and 6.2 and since $\mu_{s}^{*}(t)$ is nonincreasing on $J$,

$$
\left|z_{s}^{*}(t)\right| \leqq c_{2}\left|z_{s}^{*}\left(\tau^{*}\right)\right|+c_{2} \mu_{s}^{*}\left(\operatorname{Min}\left(t, \tau^{*}\right)\right)\left|t-\tau^{*}\right|, \quad t \in J,
$$

for sufficiently large $s$ in $P_{2}$. Since $\left|z_{s}^{*}\left(\tau^{*}\right)\right| \leqq 1$, it follows that $z_{s}^{*}(t)$ are bounded over $P_{2}$ for every fixed $t$ in $J$.

Now $0 \leqq \mu_{s}^{*}\left(\tau^{*}\right) \leqq 1$, hence $\mu_{s}^{*}\left(\operatorname{Min}\left(t, \tau^{*}\right)\right) \leqq 1+\mu_{s}^{*}(t)$ and thus

$$
\left|z_{s}^{*}(t)\right| \leqq c_{2}+c_{2}\left(t_{1}-t_{0}\right)\left(\mu_{s}^{*}(t)+1\right) \text {. }
$$

Lemma 6.4. There exists an infinite subsequence $P_{3}$ of $P_{2}$ and functions $\mu^{*}(t), z^{*}(t)$ and $v^{*}(t)$ such that $\mu_{s}^{*}(t), z_{s}^{*}(t)$ and $v_{s}^{*}(t)$ converge, for every $t$ in $T-L \cap Z$, to resp. $\mu^{*}(t), z^{*}(t)$ and $v^{*}(t)=z^{*}(t)+\mu^{*}(t) a_{x}\left(x^{*}(t)\right)$ over $P_{3}$. The function $\mu^{*}(t)$ is non-negative and nonincreasing and the function $z^{*}(t)$ is Lipschitz-continuous on every closed subinterval of $T-L \cap Z$.

Proof. Let $0<\eta<\frac{1}{2}$, let $P^{\prime}$ be an infinite subsequence of $P_{2}$ and let $J$ be maximal. Consider the closed interval $\left[\theta_{0}, \theta_{1}\right]$, where $\theta_{0}=\tau_{0}(J)+\eta\left(\tau_{1}(J)-\tau_{0}(J)\right)$ and $\theta_{1}=\tau_{1}(J)-\eta\left(\tau_{1}(J)-\tau_{0}(J)\right)$. By Lemma 6.2 and by 4.3.4, $\mu_{s}^{*}(t)$ are nonnegative and nonincreasing over $\left[\theta_{0}, \theta_{1}\right]$ for sufficiently large $s$ and, by Lemma 6.3, $\psi(t)=\limsup _{P_{2}} \mu_{s}^{*}(t)$ exists over $\left[\theta_{0}, \theta_{1}\right]$. Thus $\psi(t)$ exists and is non-negative and nonincreasing on $\left[\theta_{0}, \theta_{1}\right]$, hence $\mu_{s}^{*}(t), s$ in $P_{2}$, are nonincreasing and uniformly bounded over $\left[\theta_{0}, \theta_{1}\right]$. By Lemma 5.2,

$$
\left|z_{s}^{*}(t)\right| \leqq c_{2}+c_{2}\left|t-\tau^{*}\right| \mu_{s}^{*}\left(\operatorname{Min}\left(t, \tau^{*}\right)\right), \quad t \in\left[\theta_{0}, \theta_{1}\right]
$$

hence $z_{s}^{*}(t)$ are uniformly bounded over $\left[\theta_{0}, \theta_{1}\right]$. Furthermore, by Lemma 6.2, $z_{s}^{*}(t)$ are absolutely continuous on $\left[\theta_{0}, \theta_{1}\right]$ for sufficiently large $s$. It was observed in 4.1 that the curves $x_{s}(t), t \in T$, are contained in the compact set $D$ for all $s$; hence, by $2.2 .4,\left|f_{x}\left(x_{s}(t), t, \sigma_{s}(t)\right)\right|$ and $\left|b_{x}\left(x_{s}(t), t, \sigma_{s}(t)\right)\right|, t \in T, s=1,2, \cdots$ are uniformly bounded. It follows, by (6.1.2), that $z_{s}^{*}(t)$ satisfy a uniform Lipchitz condition over $\left[\theta_{0}, \theta_{1}\right]$ for all sufficiently large $s$.

Thus $\mu_{s}^{*}(t)$ and $z_{s}^{*}(t)$ are uniformly bounded and of uniformly bounded variation over $\left[\theta_{0}, \theta_{1}\right]$ for all $s$ in $P^{\prime}$; hence, by Helly's selection theorem, there exists an infinite subsequence $P^{\prime \prime}=P^{\prime \prime}\left(P^{\prime}, J, \eta\right)$ of $P^{\prime}$ such that $\mu_{s}^{*}(t)$ resp. $z_{s}^{*}(t)$ converge over $\left[\theta_{0}, \theta_{1}\right]$ to limit functions $\mu^{*}(t)$ resp. $z^{*}(t)$ as $s \rightarrow \infty$ over $P^{\prime \prime}$. The function $\mu^{*}(t)$ is non-negative and nonincreasing and $z^{*}(t)$ is Lipschitz-ccntinuous over $\left[\theta_{0}, \theta_{1}\right]$.

We now consider a sequence $\eta_{j}, j=1,2, \cdots$, converging to 0 . The set $T-L \cap Z$, being open relative to $T$, is a denumerable union of maximal subintervals $J_{i}, i=1,2, \cdots$. We now let $P_{1}^{1}=P_{2}$ and, recursively, $P_{j+1}^{i}=P^{\prime \prime}\left(P_{j}^{i}, J_{i}, \eta_{j}\right)$, $i, j=1,2, \cdots$, where $P_{1}^{i+1}$ is the diagonal subsequence of $P_{1}^{i}, P_{2}^{i}, \cdots$, etc. Finally, 
letting the first element of $P_{3}$ be the first one of $P_{1}^{1}$, the second element of $P_{3}$ be the second one of $P_{2}^{2}, \cdots$,etc., we conclude that $\mu_{s}^{*}(t)$ resp. $z_{s}^{*}(t)$ converge over $T-L \cap Z$, as $s \rightarrow \infty$ over $P_{3}$, to limit functions $\mu^{*}(t)$ and $z^{*}(t)$ which satisfy the statement of the lemma.

Now $v_{s}^{*}(t)=z_{s}^{*}(t)+\mu_{s}^{*}(t) a_{x}\left(x_{s}(t)\right)$ for all $s, \lim _{P} x_{s}(t)=x^{*}(t), P_{3}$ is a subsequence of $P, x_{s}(t) \in D$ for $t \in T$ and all $s$ in $P$ and $a_{x}(x)$ is uniformly continuous over compact $D$. It follows that $\lim _{P_{3}} v_{s}^{*}(t)=v^{*}(t)=z^{*}(t)+\mu^{*}(t) a_{x}\left(x^{*}(t)\right)$ over $T-L \cap Z$.

Lemma 6.5. $\left|z^{*}(t)\right|+\mu^{*}(t) \neq 0$ for $t \in T-L \cap Z$.

Proof. Assume the contrary and let $\beta\left(t^{*}\right)=\left|z^{*}\left(t^{*}\right)\right|+\mu^{*}\left(t^{*}\right)=0$ for some $t^{*}$ in $T-L \cap Z$. Let $J$ be the maximal subinterval of $T-L \cap Z$ to which $t^{*}$ belongs. It follows directly from Lemma 6.4 that $\mu^{*}(t)$ is non-negative and nonincreasing over $J$. The set $U=\left\{t \in J \mid \mu^{*}(t)=0\right\}$ is nonempty and $\theta=\inf U \leqq t^{*}$.

It follows from Lemmas 5.2 and 6.2 that

$$
\left|z^{*}(t)\right| \leqq c_{2}\left|z^{*}\left(t^{\prime}\right)\right|+c_{2}\left|\int_{t}^{t^{\prime}} \mu^{*}(\tau) d \tau\right|, \quad t \in J, t^{\prime} \in J .
$$

Now, if $\theta=\tau_{0}(J)$ then $\mu^{*}(t)=0$ for $t>\theta, t \in J$, and, by (6.5.1),

$$
\left|z^{*}\left(\tau^{*}(J)\right)\right|=\beta\left(\tau^{*}(J)\right)=1 \leqq c_{2}\left|z^{*}\left(t^{*}\right)\right|,
$$

contradicting the assumption that $\beta\left(t^{*}\right)=0$. Thus $\theta>\tau_{0}(J)$.

Let $\theta_{j}, j=1,2, \cdots$, be an increasing sequence in $J$ converging to $\theta$. Then, again by (6.5.1),

$$
\begin{aligned}
\left|z^{*}\left(\theta_{j}\right)\right| & \leqq c_{2}\left|z^{*}\left(t^{*}\right)\right|+c_{2} \int_{\theta_{j}}^{t^{*}} \mu^{*}(\tau) d \tau \\
& =c_{2} \int_{\theta_{j}}^{\theta} \mu^{*}(\tau) d \tau \leqq c_{2}\left(\theta-\theta_{j}\right) \mu^{*}\left(\theta_{j}\right) .
\end{aligned}
$$

Since $\mu^{*}\left(\theta_{j}\right) \neq 0$, we have

$$
w^{*}\left(\theta_{j}\right)=\frac{\mu^{*}\left(\theta_{j}\right)}{\left|z^{*}\left(\theta_{j}\right)\right|+\mu^{*}\left(\theta_{j}\right)} \geqq 1-c_{2}\left(\theta-\theta_{j}\right),
$$

implying $\theta \in L$.

We must also have $\theta \in Z$. Otherwise, for some positive $\delta$ and for sufficiently large $s$ in $P_{3}, a\left(x_{s}(t)\right) \neq 0$ over $[\theta-\delta, \theta+\delta] \cap J$ and, by 4.3 .4 and Lemmas 6.2 and $6.4, \mu^{*}(t)$ is constant over $[\theta-\delta, \theta+\delta] \cap J$. Since $[\theta-\delta, \theta+\delta] \cap J$ contains points of $U$ and points to the left of $\theta$, this contradicts the definition of $\theta$ as inf $U$.

Thus $\theta \in L \cap Z$, contradicting the previous result that $\tau_{0}(J)<\theta \leqq t^{*}$, hence $\theta \in J \subset T-L \cap Z$.

This completes the proof of the lemma. 
VII. The generalized Weierstrass $E$-condition.

7.1. We have defined, in Lemma 6.4 , functions $\mu^{*}(t), z^{*}(t)$ and $v^{*}(t)$ over $T-L \cap Z$. We now complete their definition by setting

(7.1.1) $\quad z^{*}(t)=o$ (the null vector), $\mu^{*}(t)=1, v^{*}(t)=a_{x}\left(x^{*}(t)\right)$ for $t \in L \cap Z$.

LEMMA 7.2. There exists a function $\sigma^{*}(t)$ from $T$ to $S$ such that

$$
\begin{aligned}
& \dot{x}^{*}(t)=f\left(x^{*}(t), t, \sigma^{*}(t)\right) \text { a.e. in } T, \\
& \dot{z}^{*}(t)=-f_{x}^{T}\left(x^{*}(t), t, \sigma^{*}(t)\right) z^{*}(t)-\mu^{*}(t) b_{x}\left(x^{*}(t), t, \sigma^{*}(t)\right) \text { a.e. in } T-L \cap Z .
\end{aligned}
$$

Furthermore, for every maximal $J, z^{*}\left(\tau_{1}(J)-0\right)=o$ if $\tau_{1}(J) \in L \cap Z$.

Proof. Let $E$ be a euclidean space, $T$ a closed interval of the real axis, $T^{\prime} \subset T,\left|T^{\prime}\right|=|T|, V$ an open set in $E, A$ a compact subset of $V$ and $F(x, t)$, $\boldsymbol{x} \in \boldsymbol{V}, \boldsymbol{t} \in \boldsymbol{T}$, a compact and convex set in $E$. Assume that $\boldsymbol{F}(\boldsymbol{x}, t)$ is uniformly bounded for $(x, t) \in A \times T^{\prime}$ and that $\boldsymbol{F}(\boldsymbol{x}, t)$ is "quasi-continuous" [1, p. 119] (or, in the language of Filippov [5, p. 76], "upper semi-continuous with respect to inclusion') at $(\boldsymbol{x}, t)$ for all $(\boldsymbol{x}, t) \in \boldsymbol{A} \times \boldsymbol{T}^{\prime}$, i.e., given any positive $\delta$, there exists a positive $\eta(\delta, x, t)$ such that $\boldsymbol{F}\left(\boldsymbol{x}^{\prime}, t^{\prime}\right)$ is contained in a $\delta$-neighborhood of $\boldsymbol{F}(\boldsymbol{x}, t)$ in $V$ provided

$$
\left|t-t^{\prime}\right|+\left|x-x^{\prime}\right| \leqq \eta(\delta, x, t) \text { and }(x, t) \in A \times T^{\prime}
$$

Let $\boldsymbol{x}_{j}(t), t \in T, j=1,2, \cdots$, be a sequence of curves, all contained in $\boldsymbol{A}$, and such that

$$
\dot{x}_{j}(t) \in F\left(x_{j}(t), t\right) \text { a.e. in } T, \quad j=1,2, \cdots .
$$

Let, finally, $x(t)$ be a uniform limit, as $j \rightarrow \infty$, of the curves $x_{j}(t), t \in T$.

It was shown in [1, Theorem 3.1, p. 119] and, effectively, in [5, Theorem 1, p. 2] that, in that case,

$$
\dot{\boldsymbol{x}}(t) \in \boldsymbol{F}(\boldsymbol{x}(t), t) \text { a.e. in } \boldsymbol{T} \text {. }
$$

Let now $\boldsymbol{F}_{j}(\boldsymbol{x}, t),(\boldsymbol{x}, t) \in \boldsymbol{A} \times \boldsymbol{T}, j=1,2, \cdots$, be convex and compact sets which converge uniformly, as $j \rightarrow \infty$, to $\boldsymbol{F}(\boldsymbol{x}, t)$ over $\boldsymbol{A} \times \boldsymbol{T}^{\prime}$ in the sense that, given any positive $\delta$, there exists $j(\delta)$ such that each of the two sets $\boldsymbol{F}_{j}(\boldsymbol{x}, t)$ and $\boldsymbol{F}(\boldsymbol{x}, t)$ is in a $\delta$-neighborhood of the other provided $j \geqq j(\delta)$. Let, furthermore, the assumption (7.2.1) be replaced by

$$
\dot{x}_{j}(t) \in F_{j}\left(x_{j}(t), t\right) \text { a.e. in } T, \quad j=1,2, \cdots .
$$

Then we can easily show that the conclusion (7.2.2) still holds. Let, indeed, $\varepsilon$ be positive and let $\boldsymbol{F}_{\varepsilon}(\boldsymbol{x}, t)$ be the $\varepsilon$-neighborhood of $\boldsymbol{F}(\boldsymbol{x}, t)$. Then there exists an integer $j(\varepsilon)$ such that $\boldsymbol{F}_{j}(\boldsymbol{x}, t) \subset \boldsymbol{F}_{\varepsilon}(\boldsymbol{x}, t)$ for $(\boldsymbol{x}, t) \in \boldsymbol{A} \times \boldsymbol{T}$ and $j \geqq j(\varepsilon)$. Thus $\dot{\boldsymbol{x}}_{j}(t) \in \boldsymbol{F}_{\imath}\left(\boldsymbol{x}_{j}(t), t\right)$ a.e. in $\boldsymbol{T}$ for $j \geqq j(\varepsilon)$, whence it follows, by the quoted arguments, 
that $\dot{x}(t) \in F_{\varepsilon}(x(t), t)$ a.e. in $\boldsymbol{T}$. Since $\varepsilon$ can be chosen arbitrarily small, relation (7.2.2) now follows.

We now apply this result as follows. Let the infinite sequence $P_{3}$ of Lemma 6.4 be $s_{1}, s_{2}, \cdots$, let $E=E_{n} \times E_{n}$, let $T$ be an arbitrary nondegenerate closed subinterval of $T-L \cap Z$ over which the functions $\mu_{s_{j}}^{*}(t), j=1,2, \cdots$ converge uniformly to $\mu^{*}(t)$, let $T^{\prime}=T \cap T^{\prime}, V=V^{\prime} \times E_{n}, D_{z}=$ Closure of $\left(\left\{z \in E_{n} \mid z=z_{s}^{*}(t)\right.\right.$ or $z=z^{*}(t)$ for some $s$ in $P_{3}$ and some $\left.\left.t \in T\right\}\right), A=D \times D_{z}, x=(x, z)$, $\boldsymbol{F}(\boldsymbol{x}, t)=\left\{(\xi, \eta) \mid \xi=f(x, t, \sigma), \eta=-f_{x}^{T}(x, t, \sigma) z-\mu^{*}(t) b_{x}(x, t, \sigma)\right.$ for some $\sigma$ in $\left.S\right\}$, $(x, z, t) \in D \times D_{z} \times \boldsymbol{T}, \boldsymbol{F}_{j}(\boldsymbol{x}, t)=\left\{(\xi, \eta) \mid \xi=f(x, t, \sigma), \eta=-f_{x}^{T}(x, t, \sigma) z-\mu_{s_{j}}^{*}(t) b_{x}(x, t, \sigma)\right.$ for some $\sigma$ in $S\}$.

Let, furthermore, $\boldsymbol{x}_{j}(t)=\left(x_{s_{j}}(t), z_{s_{j}}^{*}(t)\right), t \in T, j=1,2, \cdots$. Then it easily follows from $2.1,2.2,4.1,(6.1 .2), 6.4$ and our previous argument that

$$
\dot{\boldsymbol{x}}(t) \in \boldsymbol{F}(\boldsymbol{x}, t) \text { a.e. in } \boldsymbol{T}
$$

or, equivalently, there exists a function $\sigma^{*}(t)$ from $T \cap T^{\prime}$ to $S$ such that

$$
\begin{aligned}
& \dot{x}^{*}(t)=f\left(x^{*}(t), t, \sigma^{*}(t)\right) \text { a.e. in } T, \\
& \dot{z}^{*}(t)=-f_{x}^{T}\left(x^{*}(t), t, \sigma^{*}(t)\right) z^{*}(t)-\mu^{*}(t) b_{x}\left(x^{*}(t), t, \sigma^{*}(t)\right) \text { a.e. in } T .
\end{aligned}
$$

Since, as an easy consequence of Egoroff's theorem, $T-L \cap Z$ can be covered (except possibly for a set of measure 0), by a finite or denumerable collection of intervals like $T$, it follows that these differential equations hold a.e. in $T-L \cap Z$. We now complete the definition of $\sigma^{*}(t)$ by setting $\sigma^{*}(t)=\sigma(t), t \in L \cap Z$, where $\sigma(t)$ is as defined in (4.1.2).

Finally, it was shown in 6.1 that $w^{*}(t)-c_{2} t$ is nonincreasing over $T$, hence it follows from the definition of $L$ that, for every maximal $J$ such that $\tau_{1}(J) \in L$, we have $w^{*}\left(\tau_{1}(J)-0\right)=1$. Since, by Lemmas 6.4 and 6.5 , $w^{*}(t)=\mu^{*}(t) /\left(\left|z^{*}(t)\right|+\mu^{*}(t)\right)$ over $J$ and $\mu^{*}(t)$ is nonincreasing over $J$, it follows that $\left|z^{*}\left(\tau_{1}(J)-0\right)\right|=o$ if $\tau_{1}(J) \in L$.

This completes the proof of the lemma.

Lemma 7.3 (The generalized Weierstrass E-CONDition).

$$
v^{*}(t) \cdot f\left(x^{*}(t), t, \sigma^{*}(t)\right)=\underset{\sigma \in S}{\operatorname{Min}} v^{*}(t) \cdot f\left(x^{*}(t), t, \sigma\right) \text { a.e. in } T
$$

(where $\left.v^{*}(t)=z^{*}(t)+\mu^{*}(t) a_{x}\left(x^{*}(t)\right)\right)$.

Proof. We shall first prove that the relation holds a.e. in $T-L \cap Z$. By Lemma $6.4, z^{*}(t)$ is absolutely continuous and $\mu^{*}(t)$ is nonincreasing over every closed subinterval of $T-L \cap Z$ and, by $4.1, x^{*}(t)$ is absolutely continuous over $T$. It follows that $v^{*}(t)$ is continuous a.e. in $T-L \cap Z$. By Lemma 6.2 and 4.3.4, $\mu_{s}^{*}(t)$ is nonincreasing over every closed subinterval of $T-L \cap Z$ for sufficiently large $s$ in $P_{3}$; hence, by the inequality of Lemma $6.3, z_{s}^{*}(t)$, and consequently 
$v_{s}^{*}(t)$, are uniformly bounded over every closed subinterval of $T-L \cap Z$ for $s$ in $P_{3}$.

By 4.3.6 and 4.3.7,

$$
v_{s}^{*}(t) \cdot f\left(x_{s}(t), t, \sigma_{s}(t)\right)=v_{s}^{*}(t) \cdot \dot{x}_{s}(t)=\underset{\sigma \in S}{\operatorname{Min}} v_{s}^{*}(t) \cdot f\left(x_{s}(t), t, \sigma\right) \text { a.e. in } T
$$

for all $s$ in $P_{1}$.

Let $T_{1}$ be the subset of $T^{\prime}-L \cap Z$ over which the above relation is satisfied, over which $v^{*}(t)$ is continuous and over which $\dot{x}^{*}(t)$ exists and satisfies the first equation of Lemma 7.2. Clearly $\left|T_{1}\right|=|T-L \cap Z|$. Let $\theta \in T_{1}, \theta<t_{1}$ and let $h$ be positive and sufficiently small so that $[\theta, \theta+h] \subset T-L \cap Z$.

We have

$$
x^{*}(\theta+h)-x^{*}(\theta)=\lim _{P_{3}}\left(x_{s}(\theta+h)-x_{s}(\theta)\right)=\lim _{P_{3}} \int_{\theta}^{\theta+h} \dot{x}_{s}(\tau) d \tau ;
$$

hence,

$$
\frac{1}{h} v^{*}(\theta) \cdot\left(x^{*}(\theta+h)-x^{*}(\theta)\right)
$$

$$
=\lim _{P_{3}} \frac{1}{h} \int_{\theta}^{\theta+h}\left[v_{s}^{*}(\tau) \dot{x}_{s}(\tau)+\left(v^{*}(\tau)-v_{s}^{*}(\tau)\right) \dot{x}_{s}(\tau)+\left(v^{*}(\theta)-v^{*}(\tau)\right) \dot{x}_{s}(\tau)\right] d \tau .
$$

Now $\left|\dot{x}_{s}(\tau)\right| \leqq c_{1}$ a.e. in $T$ and $v_{s}^{*}(\tau)$ are, as shown above, uniformly bounded over $[\theta, \theta+h]$ for $s$ in $P_{3}$. By Lemma $6.4, \lim _{P_{3}} v_{s}^{*}(t)=v^{*}(t)$ over $[\theta, \theta+h]$. It follows thus from (7.3.1) and (7.3.2) that, for every $\sigma$ in $S$,

$$
\begin{aligned}
\frac{1}{h} v^{*}(\theta) \cdot\left(x^{*}(\theta+h)-x^{*}(\theta)\right) \leqq & \frac{1}{h} \int_{\theta}^{\theta+h} v^{*}(\tau) \cdot f\left(x^{*}(\tau), \tau, \sigma\right) d \tau \\
& +\frac{c_{1}}{h} \int_{\theta}^{\theta+h}\left|v^{*}(\theta)-v^{*}(\tau)\right| d \tau .
\end{aligned}
$$

By 2.2.3 and the definition of $T_{1}, v^{*}(t)$ and $f\left(x^{*}(t), t, \sigma\right)$ are continuous at $\theta$ and $x^{*}(t)$ is differentiable at $\theta$. We conclude, letting $h \rightarrow 0$, that

$$
v^{*}(\theta) \cdot f\left(x(\theta), \theta, \sigma^{*}(\theta)\right)=v^{*}(\theta) \cdot \dot{x}^{*}(\theta) \leqq v^{*}(\theta) \cdot f\left(x^{*}(\theta), \theta, \sigma\right)
$$

for every $\sigma$ in $S$ and every $\theta$ in $T_{1}$.

We must now consider the set $L \cap Z$. Since, by $6.1, w^{*}(t)-c_{2} t$ is nonincreasing, it follows easily that $w^{*}(t)=1$ a.e. in $L \cap Z$. Let now $v_{s}^{*}(t)=v_{s}(t) /\left(\left|z_{s}(t)\right|+\mu_{s}(t)\right)$ for $t \in L \cap Z$ and for $s$ in $P_{1}$. Then $\lim _{P_{3}} v_{s}^{*}(t)=a_{x}\left(x^{*}(t)\right)=v^{*}(t)$ a.e. in $L \cap Z$ and $\left|v_{s}^{*}(t)\right|$ are, for $s$ in $P_{3}$, uniformly bounded (by $\left.1+\operatorname{Max}_{x \in D}\left|a_{x}(x)\right|\right)$ over $L \cap Z$. Let $T_{2}$ be the subset of $L \cap Z$ over which $\dot{x}^{*}(t)$ exists and satisfies the first equation of Lemma 7.2, over which relation (7.3.1) is satisfied and such that 


$$
\lim _{h \rightarrow 0} \frac{1}{h}|L \cap Z \cap[\theta, \theta+h]|=1
$$

for $\theta \in T_{2}$. It is well known that the last equality holds a.e. in $L \cap Z$; hence, $\left|T_{2}\right|=|L \cap Z|$.

Let now $\theta \in T_{2}, h>0, \theta+h \in T$ and let $T_{3}=[\theta, \theta+h] \cap T_{2}$ and $T_{4}=[\theta, \theta+h]-T_{2}$. We have

$$
\frac{1}{h} v^{*}(\theta) \cdot\left(x^{*}(\theta+h)-x^{*}(\theta)\right)=\lim _{\mathbf{P}_{1}} \frac{1}{h} \int_{T_{3}}+\lim _{\boldsymbol{P}_{1}} \frac{1}{h} v^{*}(\theta) \cdot \int_{T_{4}} \dot{x}_{s}(\tau) d \tau,
$$

the integrand of the integral over $T_{3}$ being the same as on the right-hand side of (7.3.2). We then derive, in the same manner as before, the relation

$$
\begin{aligned}
\frac{1}{h} v^{*}(\theta) \cdot\left(x^{*}(\theta+h)-x^{*}(\theta)\right) & \leqq \frac{1}{h} \int_{T_{3}} v^{*}(\tau) \cdot f\left(x^{*}(\tau), \tau, \sigma\right) d \tau \\
+ & \frac{c_{1}}{h} \int_{T_{3}}\left|v^{*}(\theta)-v^{*}(\tau)\right| d \tau+\lim _{P_{1}} \frac{1}{h} v^{*}(\theta) \int_{T_{4}} \dot{x}_{s}(\tau) d \tau .
\end{aligned}
$$

Since $\lim _{h \rightarrow 0}(1 / h)\left|T_{3}\right|=1$ and $\lim _{h \rightarrow 0}(1 / h)\left|T_{4}\right|=0$, we may complete the proof in the same manner as before.

VIII. Support (transversality) conditions. Completing the proof of Theorem 3.1.

LEMMA 8.1. Either alternative 3.1.1 of Theorem 3.1 is satisfied or there exists a non-negative number $\gamma^{1}$ such that

and

$$
\left(\gamma^{1} \delta_{1}-z^{*}\left(t_{1}\right)\right) \cdot c_{1, \xi}\left(\xi_{1}^{*}\right) \xi_{1}^{*}=\underset{\xi_{1} \in C_{1}}{\operatorname{Min}}\left(\gamma^{1} \delta_{1}-z^{*}\left(t_{1}\right)\right) c_{1, \xi}\left(\xi_{1}^{*}\right) \xi_{1}
$$

$$
v^{*}\left(t_{0}\right) \cdot c_{0, \xi}\left(\xi_{0}^{*}\right) \xi_{0}^{*}=\underset{\xi_{0} \in C_{0}}{\operatorname{Min}} v^{*}\left(t_{0}\right) \cdot c_{0, \xi}\left(\xi_{0}^{*}\right) \xi_{0} .
$$

Proof. We have shown, in 5.1, that either the alternative 3.1.1 of Theorem 3.1 is satisfied or there exists an infinite sequence $P_{1}$ of integers $s$ for which the second alternative of Lemma 4.3 holds. Consider, in the latter case, the statement 4.3.9 of Lemma 4.3. If, over some infinite subsequence $P_{3}^{\prime}$ of $P_{3}, \gamma_{s}^{1} \neq 0$ and $\lim _{P_{3}^{\prime}}\left|z_{s}\left(t_{1}\right)\right| / \gamma_{s}^{1}=0$, then it follows from 4.3.9 that

where

$$
c_{1, \xi}^{1}\left(\xi_{1}^{*}\right) \xi_{1}^{*}=\operatorname{Min}_{\xi_{1} \in C_{1}} c_{1, \xi}^{1}\left(\xi_{1}^{*}\right) \xi_{1},
$$

$$
\xi_{1}^{*}=\lim _{P_{2}} \xi_{1, s}=\lim _{P_{3}^{\prime}} \xi_{1, s} \text { (see 6.1). }
$$

In this case, therefore, the alternative 3.1.1 of Theorem 3.1 is also satisfied.

In view of the above argument it remains to consider the case when the second 
alternative of Lemma 4.3 holds over an infinite sequence $P_{1}$ and, over some infinite subsequence $P_{3}^{\prime \prime}$ of $P_{3},\left|z_{s}\left(t_{1}\right)\right| \neq 0$ and $\gamma_{s}^{1} /\left|z_{s}\left(t_{1}\right)\right|$ are bounded.

If $t_{1} \in L \cap Z$ then $z^{*}\left(t_{1}\right)=o$ and relation (8.1.1) is trivially satisfied by setting $\gamma^{1}=0$. If $t_{1} \in T-L \cap Z$, let $J$ be the maximal interval to which $t_{1}$ belongs and let $\alpha_{s}=\left|z_{s}\left(\tau^{*}(J)\right)\right|+\mu_{s}\left(\tau^{*}(J)\right), \quad s$ in $P_{3}^{\prime \prime}$. Since, by 4.3.2, $\alpha_{s} \neq 0$, since $z_{s}\left(t_{1}\right) / \alpha_{s}=z_{s}^{*}\left(t_{1}\right) \rightarrow z^{*}\left(t_{1}\right)$ over $P_{3}^{\prime \prime}$ and since $\gamma_{s}^{1} /\left|z_{s}\left(t_{1}\right)\right|$ are bounded, we may find an infinite subsequence $P_{3}^{\prime \prime \prime}$ of $P_{3}^{\prime \prime}$ and a non-negative $\gamma^{1}$ such that $\lim _{P_{3}}^{\prime \prime \prime} \gamma_{s}^{1} / \alpha_{s}=\gamma^{1}$ and relation (8.1.1) now easily follows from 4.3.9.

If $t_{0} \in T-L \cap Z$ then relation (8.1.2) is derived from (4.3.8) by dividing both sides by $\left|z_{s}\left(\tau^{*}(J)\right)\right|+\mu_{s}\left(\tau^{*}(J)\right.$ ) (where $J$ is the maximal interval containing $t_{0}$ ) and passing to the limit over $P_{3}$.

If $t_{0} \in L \cap Z$ then, by the definition of $L$, either $w^{*}\left(t_{0}\right)=1$ or $\lim \sup _{\tau \rightarrow t_{0}+0} w^{*}(\tau)$ $=1$. Since, by $6.1, w^{*}(t)-c_{2} t$ is nonincreasing over $T$, it follows that $w^{*}\left(t_{0}\right)=1$, i.e., $\lim _{P_{2}} \mu_{s}\left(t_{0}\right) /\left(\left|z_{s}\left(t_{0}\right)\right|+\mu_{s}\left(t_{0}\right)\right)=1$. This implies that $\lim _{P_{2}} v_{s}\left(t_{0}\right) /\left(\left|z_{s}\left(t_{0}\right)\right|+\mu_{s}\left(t_{0}\right)\right)$ $=a_{x}\left(x^{*}\left(t_{0}\right)\right)=v^{*}\left(t_{0}\right)$. Relation (8.1.2) is now derived from (4.3.8) by dividing both sides by $\left|z_{s}\left(t_{0}\right)\right|+\mu_{s}\left(t_{0}\right)$ and passing to the limit over $P_{2}$.

8.2. Completing the proof of Theorem 3.1. Let $x(t)=x^{*}(t), t \in T$. We have shown, in 4.1 and in Lemma 4.2, that $x(t)$ exists and is a relaxed minimizing curve with respect to $a(x)$. By Assumption 2.1 and by [1, Theorem 2.2, p. 113], $x(t)$ can be uniformly approximated by solutions of the differential equations (1.1).

We shall now show that, if alternative 3.1.1 of Theorem 3.1 does not hold, then alternative 3.1.2 is satisfied by setting $M=L \cap Z, \sigma(t)=\sigma^{*}(t), \mu(t)=\mu^{*}(t)$, $z(t)=z^{*}(t), t \in T$. Statements 3.1.2.1 and 3.1.2.2 follow directly from Lemmas 6.4 and 6.5 and from the definition of $\mu^{*}(t)$ and $z^{*}(t)$ on $L \cap Z$. By statement 4.3.4 of Lemma $4.3, \mu_{s}(t)$ are, for all $s$ in $P_{3}$, constant over every subinterval of $T-Z_{s}$ and $\mu_{s}\left(t_{1}\right)=0$. Now, we easily verify that every closed subinterval of $T-Z$ is contained in $T-Z_{s} \subset T-L_{s}$ for all sufficiently large $s$ in $P_{2}$ and in $P_{3}$. Thus, $\mu^{*}(t)$ is constant on every closed subinterval of $T-Z$. Statement 3.1.2.3 now follows from Lemma 6.4.

Statements 3.1.2.4, 3.1.2.5 and 3.1.2.6 follow from Lemmas 7.2, 7.3 and 8.1, respectively.

8.2.1. Proof of statement 3.1.2.7. We have just shown that either alternative 3.1.1 of Theorem 3.1 holds or alternative 3.1.2 is satisfied through 3.1.2.6. Assume now that alternative 3.1.1 does not hold nor is 3.1.2.7.1 satisfied.

Let $\mathscr{A}$ be the set of points $\theta$ in $T$ with the property that

$$
\begin{aligned}
& |a(x(\theta))|+\left|a_{x}(x(\theta))\right| \neq 0, \\
& z(\theta) \neq-\bar{\gamma} a_{x}(x(\theta)) \text { for all } \bar{\gamma} \geqq \mu(\theta) \text { if } \theta \in Z, \\
& \left|z(\theta)+\mu(\theta) a_{x}(x(\theta))\right| \neq 0 \text { if } \theta \in T-Z .
\end{aligned}
$$

We shall show that $\mathscr{A}$ contains $t_{1}$, that for every $\theta, \theta \in \mathscr{A} \cap Z, \theta>t_{0}$, there 
exists a positive $\varepsilon(\theta)$ such that $[\theta-\varepsilon(\theta), \theta] \subset \mathscr{A}$ and that every subinterval $K$ of $T-Z$ either belongs to $\mathscr{A}$ or has no points in common with $\mathscr{A}$. Then, letting $t_{0}^{*}=t_{0}$ if $\mathscr{A}=T$ and $t_{0}^{*}=\sup (T-\mathscr{A})$ if $\mathscr{A} \neq T$, we can easily verify that statement 3.1.2.7.2 follows.

We now proceed to prove the assertion about $\mathscr{A}$ which we have just stated.

8.2.1.1. $t_{1} \in \mathscr{A}$.

We have $\left|a\left(x\left(t_{1}\right)\right)\right|+\left|a_{x}\left(x\left(t_{1}\right)\right)\right| \neq 0$ since, otherwise, 3.1.2.7.1 would be trivially satisfied with $\gamma^{a}=0$. If $t_{1} \in T-Z$, then, by 3.1.2.1 and 3.1.2.3, $\left|z\left(t_{1}\right)+\mu\left(t_{1}\right) a_{x}\left(x\left(t_{1}\right)\right)\right|=\left|z\left(t_{1}\right)\right| \neq 0$. If $t_{1} \in Z$, assume, by way of contradiction, that $z\left(t_{1}\right)=-\bar{\gamma} a_{x}\left(t_{1}\right)$ for some $\bar{\gamma} \geqq \mu\left(t_{1}\right) \geqq 0$. We cannot have $\bar{\gamma}=0$ since, by 3.1.2.1, $\left|z\left(t_{1}\right)\right|+\mu\left(t_{1}\right)>0$. We cannot have $\bar{\gamma}>0$ either, since then, by 3.1.2.6, the statement 3.1.2.7.1 is satisfied (with $\gamma^{a}=\gamma^{1} / \bar{\gamma}$ ), contrary to assumption. Thus $t_{1} \in \mathscr{A}$.

8.2.1.2. Let $K$ be a subinterval of $T-Z$. Then $K \subset \mathscr{A}$ or $K \cap \mathscr{A}$ is empty.

By 3.1.2.3, $\mu(t)$ is constant on $K$. Let $v(t)=z(t)+\mu(t) a_{x}(x(t)), t \in K$, and assume first that $K$ is closed. Then, by 3.1.2.2, $v(t)$ is absolutely continuous on $K$. It follows now easily from 3.1.2.4 that

$$
\dot{v}(t)=-f_{x}^{T}(x(t), t, \sigma(t)) v(t) \text { a.e. in } K,
$$

hence $v(t)$ either vanishes at every point of $K$ or $|v(t)| \neq 0$ for every $t$ in $K$. Thus $K \subset \mathscr{A}$ or $K \cap \mathscr{A}$ is empty.

Since the last statement is true for any closed subinterval $K$ of $T-Z$, it remains true for any subinterval (convex subset) of $T-Z$.

8.2.1.3. Let $\theta \in(Z-L) \cap \mathscr{A}, \theta>t_{0}$. Then $[\theta-\varepsilon(\theta), \theta] \subset \mathscr{A}$ for some $\varepsilon(\theta)>0$.

Let $J$ be the maximal subinterval of $T-L \cap Z$ to which $\theta$ belongs. Since $\left|a_{x}(x(t))\right|$ is continuous and $\left|a_{x}(x(\theta))\right| \neq 0$, there exists $\varepsilon^{\prime}=\varepsilon^{\prime}(\theta)>0$ such that $\left|a_{x}(x(t))\right| \neq 0$ on $\left[\theta-\varepsilon^{\prime}, \theta\right] \subset J$. If, for some $\varepsilon^{\prime \prime}, 0<\varepsilon^{\prime \prime}<\varepsilon^{\prime},\left[\theta-\varepsilon^{\prime \prime}, \theta\right) \subset T-Z$ then, by 3.1.2.2, $|v(\theta-0)|=0$ implies $z(\theta)=-\mu(\theta-0) a_{x}(x(\theta))$. Since, by 3.1.2.3, $\mu(t)$ is nonincreasing on $J$, this contradicts $\theta \in Z \cap \mathscr{A}$. Thus $\left[\theta-\varepsilon^{\prime \prime}, \theta\right] \subset \mathscr{A}$.

It follows that if $\theta_{j}^{\prime}, j=1,2, \cdots$, is an increasing sequence in $J$ such that $\theta_{j}^{\prime} \in T-Z-\mathscr{A}, j=1,2, \cdots$, and $\theta_{j}^{\prime} \rightarrow \theta$ then there exists an increasing sequence $\theta_{j}, j=1,2, \cdots$, such that $\theta_{j} \in Z-\mathscr{A}, j=1,2, \cdots$, and $\theta_{j} \rightarrow \theta$. Assume, therefore, by way of contradiction, that such a sequence $\theta_{j}$ exists in $\left[\theta-\varepsilon^{\prime}, \theta\right]$, hence that there exist $\bar{\gamma}_{j} \geqq \mu\left(\theta_{j}\right), j=1,2, \cdots$, such that $z\left(\theta_{j}\right)=-\bar{\gamma}_{j} a_{x}\left(x\left(\theta_{j}\right)\right), j=1,2, \cdots$.

By 3.1.2.2 and the continuity of $a_{x}(x(t)), z\left(\theta_{j}\right) \rightarrow z(\theta)$ and $a_{x}\left(x\left(\theta_{j}\right)\right) \rightarrow a_{x}(x(\theta))$. The non-negative $\bar{\gamma}_{j}$ must be bounded, since, otherwise, $\left|a_{x}(x(\theta))\right|$ $=\lim _{j \rightarrow \infty}\left|a_{x}\left(x\left(\theta_{j}\right)\right)\right|=0$, contradicting $\theta \in \mathscr{A} \cap Z$. Thus $\gamma^{*}=\lim \bar{\gamma}_{j}$ exists and we have $z(\theta)=-\gamma^{*} a_{x}(x(\theta))$. Since $\bar{\gamma}_{j} \geqq \mu\left(\theta_{j}\right)$ and $\mu(t)$ is nonincreasing on $J$. have $\gamma^{*} \geqq \mu(\theta)$, thus contradicting $\theta \in \mathscr{A} \cap Z$. 
It follows that there exists $\varepsilon(\theta)>0$ such that $[\theta-\varepsilon(\theta), \theta] \subset \mathscr{A}$.

8.2.1.4. Let $\theta \in L \cap Z \cap \mathscr{A}, \theta>t_{0}$. Then $[\theta-\varepsilon(\theta), \theta] \subset \mathscr{A}$ for some $\varepsilon(\theta)>0$.

Since $\theta \in \mathscr{A} \cap Z$, there exists a positive $\varepsilon^{\prime}=\varepsilon^{\prime}(\theta)$ such that $\left|a_{x}(x(t))\right|>\frac{1}{2}\left|a_{x}(x(\theta))\right|>0$ for $\theta-\varepsilon^{\prime} \leqq t \leqq \theta$. Let $\varepsilon=\varepsilon(\theta)=\operatorname{Min}\left(\varepsilon^{\prime},\left|a_{x}(x(\theta))\right| / 4 c_{2}\right)$, where $c_{2}$ is as defined in Lemma 5.2. Let $\theta-\varepsilon \leqq t^{*} \leqq \theta$.

If $t^{*} \in L \cap Z$ then $z\left(t^{*}\right)=o \neq-\bar{\gamma} a_{x}\left(x\left(t^{*}\right)\right)$ for all $\bar{\gamma} \geqq \mu\left(t^{*}\right)=1$.

If $t^{*} \in T-L \cap Z$, let $J$ be the maximal subinterval of $T-L \cap Z$ to which $t^{*}$ belongs. It follows easily from 3.1.2.4 (or from Lemmas 5.2 and 6.2; see (6.5.1)) that

$$
|z(t)| \leqq c_{2}\left|z\left(t^{\prime}\right)\right|+c_{2} \int_{t}^{t^{\prime}} \mu(\tau) d \tau \leqq c_{2}\left|z\left(t^{\prime}\right)\right|+c_{2}\left(t-t^{\prime}\right) \mu(t)
$$

for $t \in J, t^{\prime} \in J, t<t^{\prime}$.

We have, by 3.1.2.4, $z\left(\tau_{1}(J)-0\right)=o$. Since $\theta \in L \cap Z$, we have $\tau_{1}(J) \leqq \theta$. It follows thus that

$$
\left|z\left(t^{*}\right)\right| \leqq c_{2}\left(\tau_{1}(J)-t^{*}\right) \mu\left(t^{*}\right) \leqq c_{2} \varepsilon \mu\left(t^{*}\right) \leqq \frac{1}{2}\left|a_{x}\left(x\left(t^{*}\right)\right)\right|\left|\mu\left(t^{*}\right)\right| .
$$

By 3.1.2.1, $\mu\left(t^{\#}\right)>0$, hence

$$
z\left(t^{*}\right) \neq-\bar{\gamma} a_{x}\left(x\left(t^{*}\right)\right) \text { for all } \bar{\gamma} \geqq \mu\left(t^{*}\right) .
$$

This completes the proof of 3.1.2.7.

8.2.2. Proof of statement 3.1.2.8. Assume, by way of contradiction, that the assumption of 3.1.2.8 is satisfied and that there exists a point $\theta \in M, \theta>t_{0}$. Since $M=L \cap Z \subset Z$, it follows from our assumption that there exists a number $\varepsilon, 0<\varepsilon<\theta-t_{0}$, such that

$$
\left|a_{x}(x(t))\right| \geqq \frac{1}{2}\left|a_{x}(x(\theta))\right|>0 \text { and } \operatorname{Min}_{\sigma \in S} a_{x}(x(t)) \cdot f(x(t), \tau, \sigma) \leqq \frac{1}{2} \beta
$$

for all $\tau \in T$ and $\theta-\varepsilon \leqq t \leqq \theta$.

If $[\theta-\varepsilon, \theta] \subset M$, then, by 3.1.2.4, 3.1.2.5 and (8.2.2.1), $(d / d t) a(x(t))=a_{x}(x(t)) \cdot f(x(t), t, \sigma(t))=\operatorname{Min}_{\sigma \in S} a_{x}(x(t)) \cdot f(x(t), t, \sigma) \leqq \frac{1}{2} \beta$ a.e. in $[\theta-\varepsilon, \theta]$, contradicting $[\theta-\varepsilon, \theta] \subset M \subset Z$. It follows that there exists a point $\theta^{\prime}$ in $[\theta-\varepsilon, \theta] \cap(T-M)$.

Let $J$ be the maximal interval to which $\theta^{\prime}$ belongs. Then $\tau_{1}(J) \leqq \theta$ and $\tau_{1}(J) \in M$. Since, by $6.1, w^{*}(t)-c_{2} t$ is nonincreasing over $T$, we have $w^{*}\left(\tau_{1}(J)-0\right)=1$. By 3.1.2.1, $|z(t)|+\mu(t)>0$ for all $t \in T$; hence $w^{*}(t)=\lim _{P_{3}} \mu_{s}^{*}(t) /\left(\left|z_{s}^{*}(t)\right|+\mu_{s}^{*}(t)\right)$ $=\mu(t) /(|z(t)|+\mu(t))$ for $t \in J$. We conclude that $\zeta(t)=z(t) /(|z(t)|+\mu(t))$ $+w^{*}(t) a_{x}(x(t))$ converges to $a_{x}\left(x\left(\tau_{1}(J)\right)\right)$ as $t \rightarrow \tau_{1}(J), t \in J$.

We have, by 3.1.2.4 and 3.1.2.5, $\zeta(t) \cdot f(x(t), \sigma(t))=\operatorname{Min}_{\sigma \in S} \zeta(t) \cdot f(x(t), t, \sigma)$ a.e. in $[\theta-\varepsilon, \theta]$. Since $\zeta\left(\tau_{1}(J)-0\right)=a_{x}\left(x\left(\tau_{1}(J)\right)\right)$, it follows that there exists a number 
$\varepsilon^{\prime}>0$ such that $\left[\tau_{1}(J)-\varepsilon^{\prime}, \tau_{1}(J)\right] \subset[\theta-\varepsilon, \theta]$ and $\left|\zeta(t)-a_{x}(x(t))\right| \leqq(1 / 8)|\beta| / c_{1}$ on $\left[\tau_{1}(J)-\varepsilon^{\prime}, \tau_{1}(J)\right]$; hence, by $2.2 .4,3.1 .2 .4,3.1 .2 .5$ and (8.2.2.1), $(d / d t) a(x(t))=a_{x}(x(t)) \cdot f(x(t), t, \sigma(t)) \leqq \zeta(t) \cdot f(x(t), t, \sigma(t))-(1 / 8) \beta$ $=\operatorname{Min}_{\sigma \in S} \zeta(t) \cdot f(x(t), t, \sigma)-1(/ 8) \gamma \leqq \operatorname{Min}_{\sigma \in S} a_{x}(x(t)) \cdot f(x(t), t, \sigma)-(1 / 4) \beta$ $\leqq(1 / 4) \beta<0$ a.e. on $\left[\tau_{1}(J)-\varepsilon^{\prime}, \tau_{1}(J)\right]$. This contradicts $a(x(t)) \leqq 0$ over $\left[\tau_{1}(J)-\varepsilon^{\prime}, \tau_{1}(J)\right]$ and $a\left(x\left(\tau_{1}(J)\right)\right)=0$.

This completes the proof of statement 3.1.2.8 and of Theorem 3.1.

\section{REFERENCES}

1. J. Warga, Relaxed variational problems, J. Math. Anal. Appl. 4 (1962), 111-128.

2. - Necessary conditions for minimum in relaxed variational problems, J. Math. Anal. Appl. 4 (1962), 129-145.

3. L. C. Young, Generalized curves and the existence of an attained absolute minimum in the calculus of variations, C. R. Soc. Sci. Lettres Varsovie Cl. III 30 (1937), 212-234.

4. E. J. McShane, Generalized curves, Duke Math. J. 6 (1940), 513-536.

5. A. F. Filippov, On certain questions in the theory of optimal control, J. Soc. Indust. Appl. Math. Control Ser. A 1 (1962), 76-84. (Translated by L. W. Neustadt, Vestnik Moskov. Univ. Ser. Mat. Meh. Astronom. Fiz. Him. (1959), 25-32.)

6. R. V. Gamkrelidze, Optimum-rate processes with bounded phase coordinates, Dokl. Akad. Nauk SSSR 125 (1959), 475-478.

7. L. S. Pontryagin, V. G. Boltyanskii, R. V. Gamkıelidze and E. F. Mishchenko, The mathematical theory of optimal processes, Interscience, New York, 1962.

8. L. C. Young, Necessary conditions in the calculus of variations, Acta Math. 69 (1938), 239-258.

9. E. J. McShane, Necessary conditions in generalized-curve problems of the calculus of variations, Duke Matb. J. 7 (1940), 1-27.

10. V. G. Boltyanskii, R. V. Gamkrelidze and L. S. Pontryagin, The theory of optimum processes, Dokl. Akad. Nauk SSSR 110 (1956), 7-10.

Avco Research and Advanced Development Division,

Wilmington, MASSACHUSETTS 